\title{
Stability of the hybrid epithelial/mesenchymal phenotype
}

\author{
Mohit Kumar Jolly, ${ }^{1,2}$, Satyendra C. Tripathi, ${ }^{8, *}$, Dongya Jiaa,5,*, Steven M. Mooney ${ }^{7}$, \\ Muge Celiktas ${ }^{8}$, Samir M. Hanash ${ }^{8,10}$, Sendurai A. Mani ${ }^{911}$, Kenneth J. Pienta ${ }^{12}$, \\ Eshel Ben-Jacob ${ }^{1,5,6, * *}$ and Herbert Levine ${ }^{1,2,3,4}$ \\ ${ }^{1}$ Center for Theoretical Biological Physics, Rice University, Houston, TX, USA \\ 2 Department of Bioengineering, Rice University, Houston, TX, USA \\ ${ }^{3}$ Department of Physics and Astronomy, Rice University, Houston, TX, USA \\ ${ }^{4}$ Department of Biosciences, Rice University, Houston, TX, USA \\ ${ }^{5}$ Graduate Program in Systems, Synthetic and Physical Biology, Rice University, Houston, TX, USA \\ ${ }^{6}$ School of Physics and Astronomy and The Sagol School of Neuroscience, Tel-Aviv University, Tel-Aviv, Israel \\ 7 Department of Biology, University of Waterloo, Waterloo, ON, Canada \\ ${ }^{8}$ Department of Clinical Cancer Prevention, University of Texas MD Anderson Cancer Center, Houston, TX, USA \\ ${ }^{9}$ Department of Translational Molecular Pathology, University of Texas MD Anderson Cancer Center, Houston, TX, USA \\ 10 Red and Charline McCombs Institute for the Early Detection and Treatment of Cancer, University of Texas MD Anderson \\ Cancer Center, Houston, TX, USA \\ ${ }^{11}$ Metastasis Research Center, University of Texas MD Anderson Cancer Center, Houston, TX, USA \\ 12 The James Brady Urological Institute, and Departments of Urology, Oncology, Pharmacology and Molecular Sciences, \\ Johns Hopkins School of Medicine, Baltimore, MD, USA \\ * These authors have contributed equally to this work \\ *** Deceased on June 5, 2015 \\ Correspondence to: Herbert Levine, email: herbert.levine@rice.edu \\ Keywords: partial EMT, epithelial-mesenchymal transition, cancer stem cells, multistability, cell-fate decisions \\ Received: January 08, $2016 \quad$ Accepted: March 07, 2016 \\ Published: March 17, 2016
}

\section{ABSTRACT}

Epithelial-to-Mesenchymal Transition (EMT) and its reverse - Mesenchymal to Epithelial Transition (MET) - are hallmarks of cellular plasticity during embryonic development and cancer metastasis. During EMT, epithelial cells lose cell-cell adhesion and gain migratory and invasive traits either partially or completely, leading to a hybrid epithelial/mesenchymal (hybrid E/M) or a mesenchymal phenotype respectively. Mesenchymal cells move individually, but hybrid $\mathrm{E} / \mathrm{M}$ cells migrate collectively as observed during gastrulation, wound healing, and the formation of tumor clusters detected as Circulating Tumor Cells (CTCs). Typically, the hybrid E/M phenotype has largely been tacitly assumed to be transient and 'metastable'. Here, we identify certain 'phenotypic stability factors' (PSFs) such as GRHL2 that couple to the core EMT decision-making circuit (miR-200/ZEB) and stabilize hybrid E/M phenotype. Further, we show that $\mathrm{H} 1975$ lung cancer cells can display a stable hybrid $\mathrm{E} / \mathrm{M}$ phenotype and migrate collectively, a behavior that is impaired by knockdown of GRHL2 and another previously identified PSF - OVOL. In addition, our computational model predicts that GRHL2 can also associate hybrid E/M phenotype with high tumorinitiating potential, a prediction strengthened by the observation that the higher levels of these PSFs may be predictive of poor patient outcome. Finally, based on these specific examples, we deduce certain network motifs that can stabilize the hybrid E/M phenotype. Our results suggest that partial EMT, i.e. a hybrid E/M phenotype, need not be 'metastable', and strengthen the emerging notion that partial EMT, but not necessarily a complete EMT, is associated with aggressive tumor progression. 


\section{INTRODUCTION}

During metastasis, cancer cells leave the primary tumor, travel through the circulation, and seed secondary tumors in distant organs. Metastasis can be fueled by the engines of cellular plasticity - bidirectional transitions between the epithelial and mesenchymal phenotypes the Epithelial to Mesenchymal Transition (EMT) and its reverse the Mesenchymal to Epithelial Transition (MET) [1]. EMT and MET are not specific to cancer metastasis, rather they are fundamental developmental phenomena that get aberrantly activated in cancer metastasis [1]. Cells undergoing EMT lose their cell-cell adhesion and gain migratory and invasive traits to invade the basement membrane and enter the blood vessels as Circulating Tumor Cells (CTCs) [2]. These CTCs exit at distant organs and usually undergo MET to settle down and proliferate in order to form secondary tumors during metastasis [1]. Elucidating the principles of this cellular plasticity is expected to offer crucial clues for halting metastatic progression.

While transitioning between the $\mathrm{E}$ and $\mathrm{M}$ phenotypes, cells can adopt a hybrid epithelial/ mesenchymal (E/M) or a partial EMT phenotype. Cells in this phenotype have a mix of epithelial (e.g. cell-cell adhesion) and mesenchymal (e.g. migration) traits that enable them to move collectively during mammary gland formation, trachea development, and wound healing [3, 4]. Such collective migration obviates the need for all cells to identify and respond to an external signal, and allows maximum cellular plasticity [3]. Furthermore, during metastasis, collective migration as CTC clusters has observed in the patients with lung, prostate, and breast cancer $[5,6]$. Cells in CTC clusters can enter and exit the bloodstream more efficiently [7], are resistant to anoikis [6], and form up to 50-times more tumors as compared to CTCs that have undergone a complete EMT and move individually [6]. In addition, the implications of hybrid E/M phenotype in metastasis might help explain recent studies $[8,9]$ showing that preventing cells from switching to being fully mesenchymal does not drastically affect metastasis [10]. Furthermore, the predominance of cells co-expressing $\mathrm{E}$ and $\mathrm{M}$ markers in many aggressive tumors such as melanomas and basal-like and claudin-low breast cancers argues for a strong association of the hybrid E/M phenotype with aggressiveness [5]. Such co-expression, as compared to the expression of $\mathrm{M}$ genes only, correlates with increased invasive and metastatic potential and predicts poor outcomes independent of the breast cancer subtype [11]. Thus, the hybrid E/M phenotype can pose a higher metastatic risk in patients as compared to the pure $\mathrm{M}$, i.e. complete EMT phenotype [5, 11].

Despite its critical significance in both physiological and pathological EMT, the hybrid E/M phenotype has not been comprehensively characterized. It has been proposed to be a 'metastable' phenotype (i.e. the cells cannot maintain this phenotype stably for a long time) that can be acquired only transiently en route to EMT $[12,13]$. But recent experiments in mammary gland development show that the cells in terminal end buds (TEBs) can be maintained in a hybrid E/M phenotype and prevented from undergoing a complete EMT by transcription factor OVOL [14]. OVOL, thus, acts as a 'phenotypic stability factor' (PSF) [15] as proposed by recent attempts to mathematically model its role in mediating EMT/MET $[16,17]$. Furthermore, OVOL not only stabilizes the hybrid E/M phenotype, but also has been predicted by mathematical models to enable the hybrid $\mathrm{E} / \mathrm{M}$ cells to gain stemness [18], i.e. regenerative and self-renewal potential during developmental EMT, and the potential to seed secondary tumors during metastasis. These observations provide the motivation to identify more such 'phenotypic stability factors' [15].

Here, via mathematical modeling, we identify two additional phenotypic stability factors that can expand the existence of the hybrid E/M phenotype - transcription factor GRHL2 and microRNA miR-145. Further, we show experimentally that partial EMT phenotype can be observed stably at a single-cell level in H1975 lung cancer cells in vitro, and knockdown of OVOL and GRHL2 can impair collective cell migration - a hallmark of the hybrid E/M phenotype - and drive a complete EMT. Finally, generalizing these findings by using a network topologybased approach, we propose several network motifs that can be utilized to identify the molecular players that can maintain a hybrid E/M phenotype and potentially are also likely to ascribe an enhanced tumor-initiating potential to the hybrid E/M phenotype.

\section{RESULTS}

\section{Partial EMT can be stably maintained at a single- cell level in vitro}

We characterized three lung adenocarcinoma cell lines H1299, H2291, and H1975 for their EMT status by immunofluorescence (IF) staining with canonical EMT markers - CDH1 (E-cadherin) and VIM (Vimentin). H1299, categorized as a mesenchymal cell line [19], stained only for Vimentin; however, H2291 and H1975, both categorized as E/M cell lines based on ensemble measurements [19], stained for both VIM and CDH1. H2291 contained subpopulations of E (cells staining only for CDH1) and $\mathrm{M}$ (cells staining only for VIM) (Figure S3A), but H1975 cells co-stained for both VIM and CDH1 individually (Figure 1A), indicating a hybrid E/M or partial EMT phenotype at a single-cell level. Similar co-expression of E and M markers at a single-cell level has been observed in multiple cell lines belonging to breast cancer [11, 17] and lung cancer [20], 
as well as in metastatic brain tumors [21], CTCs(22), and tumor buds - clusters of 1-5 malignant cells observed at the invasive front of the tumor [23]. Importantly, H1975 cells maintained this partial EMT phenotype at a single-cell level for over two months in culture, thereby demonstrating a stable phenotype (Figure S4).

A partial EMT phenotype has been implicated in collective cell migration during embryonic development and wound healing [3, 4, 12]; therefore, we conducted a scratch assay for H1299 and H1975 cell lines. H1299 cells moved largely as single cells, but H1975 cells filled the wound moving collectively and forming finger-like projections (Figure 1B). These finger-like projections are the hallmarks of collective migration as noted earlier for migration of TEBs [14], and suggest that collective cell migration as sheets or clusters might be observed in tumors as well, as also reflected by tumor budding [23] and the migration of CTCs in clusters that are formed before entering the circulation [24].
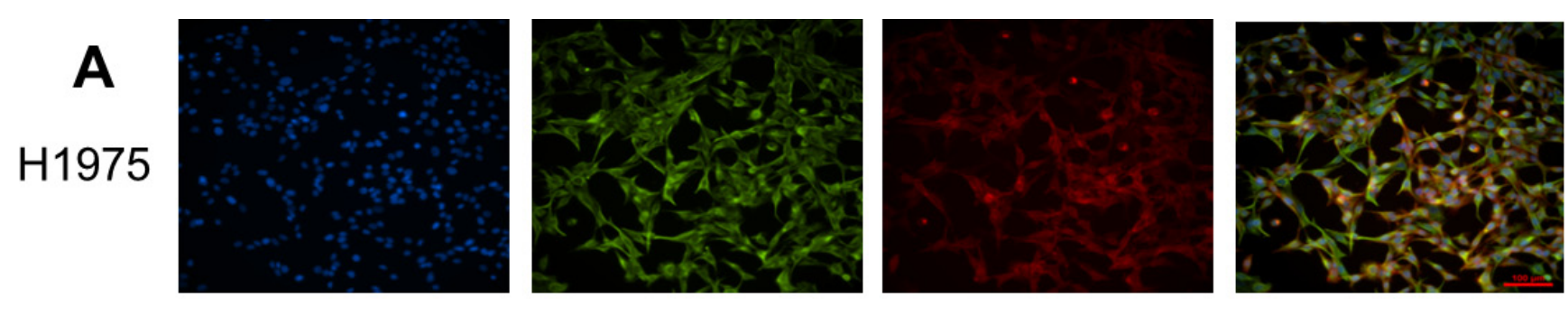

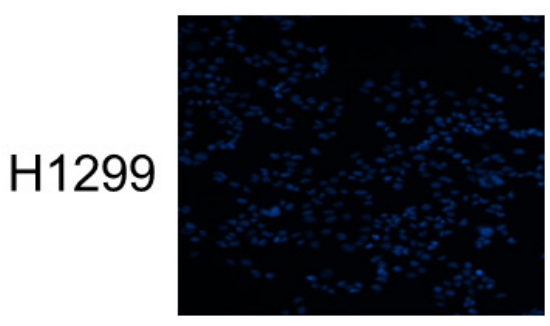

DAPI

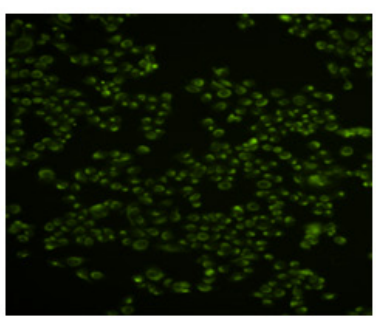

VIM

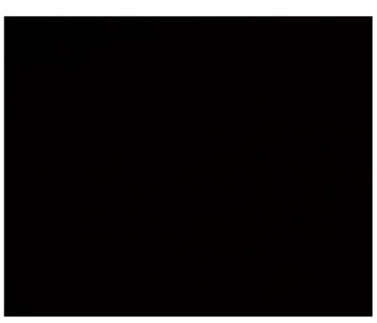

$\mathrm{CDH} 1$

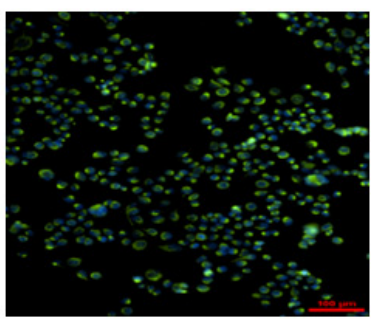

Merged

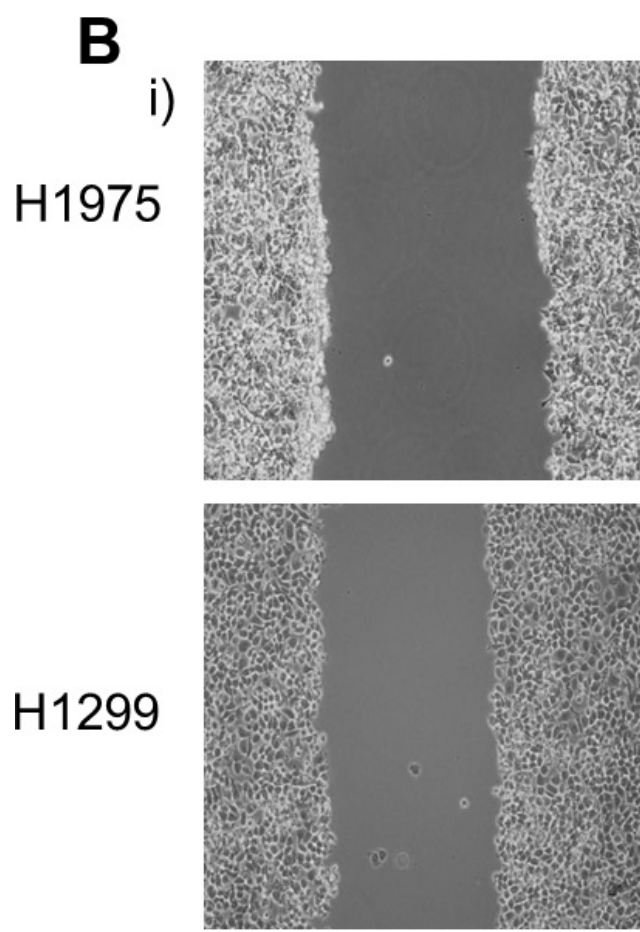

ii)
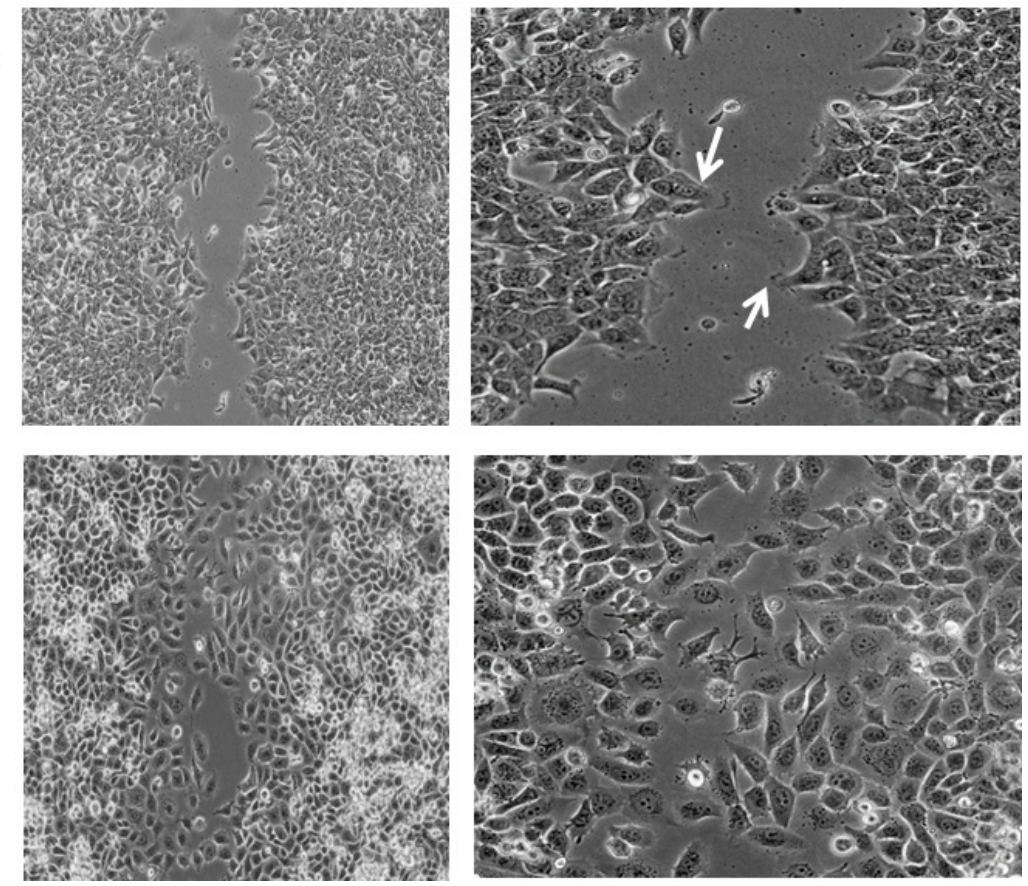

Figure 1: Characterizing the partial EMT phenotype. A. Expression of CDH1 (E-cadherin) and VIM (Vimentin) examined by immunofluorescence staining. Scale bar $100 \mu \mathrm{m}$. B. Scratch assay of H1975 and H1299 depicting different cell motility patterns. White arrows denote finger-like projections seen in H1975 cells. Panel i) shows the condition at the beginning of scratch assay ( $t=0$ hours) whereas ii) shows for $t=12$ hours for H1299 and $t=16$ hours for H1975 cells. 


\section{Mathematical modeling predicts GRHL2 and miR-145 act as 'phenotypic stability factors' for the partial EMT phenotype}

Previous experimental and theoretical analysis shows that the transcription factor OVOL can play a crucial role in maintaining a hybrid $\mathrm{E} / \mathrm{M}$ phenotype [14, $16,17]$. Here, we investigate other similar 'phenotypic stability factors' (PSFs) that have been proposed to (a) maintain collective cell migration, or (b) induce a partial EMT, or (c) couple to the 'motor of cellular plasticity' - a double negative feedback loop between miR-200 and ZEB that regulates EMT/MET in multiple tumors [25] - in a similar manner as that of OVOL (Figure 2; SI sections 1,2). First, GRHL2 - a well-known regulator of morphogenesis that controls the differentiation and migration of epithelial cell layers - can maintain collective cell migration at its endogenous levels, drives MET when overexpressed, and enables EMT when knocked down in breast cancer cells [26, 27]. Second, miR-145 has been shown to induce a partial EMT in prostate cancer cells [28], and similar to OVOL and GRHL2, can drive MET when overexpressed [28]. All of these factors - OVOL, GRHL2, and miR-145 - couple to the (miR-200/ZEB) loop that has been proposed to act as a three-way switch enabling three phenotypes - E (high miR-200,low ZEB), M (low miR-200, high ZEB), and hybrid E/M (medium miR-200, medium ZEB) [29].

As a first step towards elucidating the effect of these factors on the epithelial-hybrid-mesenchymal transition, we investigate their coupling with (miR-200/ZEB), and evaluate the response of the coupled circuits to different levels of SNAIL, an input to the (miR-200/ZEB) circuit, via a mathematical model that considers the dynamics of miR-200, ZEB mRNA, ZEB protein, and GRHL2 protein as a function of their regulatory interactions, and treats SNAIL as a driving input to this circuit.

The response of the different circuits to varying levels of SNAIL (mimicking the effect of EMT-inducing signals) is presented as a bifurcation diagram of ZEB mRNA levels in Figure 3. Lower levels ( $<100$ molecules) of ZEB mRNA denote an epithelial (E) phenotype, intermediate values ( $\sim 200-450$ molecules) correspond to a hybrid E/M state and higher values denote a mesenchymal (M) phenotype (blue solid lines in Figure 3). For low SNAIL levels, cells attain only the E phenotype. With increase in SNAIL levels, EMT is induced partially hence cells attain a hybrid E/M phenotype, and further increases in SNAIL levels induce a complete EMT and consequently cells attain a mesenchymal (M) phenotype. The range of SNAIL values for which the hybrid E/M phenotype exists is much larger for the (miR-200/ZEB/GRHL2) circuit as compared to that for the (miR-200/ZEB) circuit (i.e. without being coupled with GRHL2) (compare the green area in Figure 3B vs that in Figure 3A). Most importantly, there is now a range for which the only possible state is partial EMT (region in the dotted rectangle), and therefore, in this range, the hybrid E/M phenotype is absolutely stable. In other words, after incorporating the effect of GRHL2, a parameter regime (physiological conditions) under which most cells will attain a stable hybrid E/M phenotype is enabled. Equivalently, if the cells present in the parameter region corresponding to the dotted region in Figure 3 will be sorted by flow cytometry (FACS), most of them will express both $\mathrm{E}$ and $\mathrm{M}$ markers, thereby corresponding to a hybrid E/M phenotype. A similar set of results is also observed for the (miR-200/ZEB/miR145/OCT4) circuit (compare the green area in Figure 3C $v s$ that in Figure 3A), thereby capturing the role of the 'phenotypic stability factors' (PSFs) GRHL2 and miR-145 in stabilizing the hybrid E/M phenotype.

Next, we investigated the effect of overexpression and inhibition of these 'phenotypic stability factors' by considering an external activating (SA) or inhibiting signal (SI) on GRHL2. Our model predicts that similar to the results obtained for OVOL [16], knockdown of GRHL2 and miR-145 destabilizes the hybrid E/M phenotype (note the absence of the region bound by dotted rectangle in case of SI $\neq 0$ in Figure 3D) and can lead to a complete EMT(note the much lower levels of SNAIL needed to induce a partial or complete EMT when SI $\neq 0$ in Figure 3D), and that their overexpression can induce an MET (note the much higher level of SNAIL needed to induce a partial EMT when $\mathrm{SA} \neq 0$ in Figure 3E) (also see Figure S5). These predictions are consistent with experiments showing that (a) knockdown of OVOL and GRHL2 impairs collective cell migration during mammary morphogenesis and lung development respectively [14, 27], (b) overexpression of GRHL2 and OVOL drives MET in MDA-MB-231 and PC3 cells respectively [26, 30], and (c) overexpression of miR-145 represses EMT in PC3 and DU145 cells in vitro [28], and the aggressiveness of PC3 cells in the bone in vivo [31].

\section{Experiments demonstrate that knockdown of OVOL and GRHL2 leads to a complete EMT}

To directly test our prediction that knockdown of these PSFs can drive a complete EMT, we investigated the effect of silencing OVOL2 and GRHL2 in H1975 cells, which exhibit a stable hybrid $\mathrm{E} / \mathrm{M}$ phenotype, via siRNAs. We observed that on treatment with siRNAs against GRHL2 and OVOL2, the collective migration of H1975 cells was disrupted, and they migrated more individually, suggesting the completion of EMT (Figure 4A). Consistently, cells lost E-cad (CDH1) expression both at the transcriptional and the translational level (Figure 4B, 4C) and gained ZEB1 (Figure S6). Disruption of collective migration was more pronounced in cells treated with siGRHL2, possibly because of the crucial role of GRHL2 in lung development [27]. Knockdown 

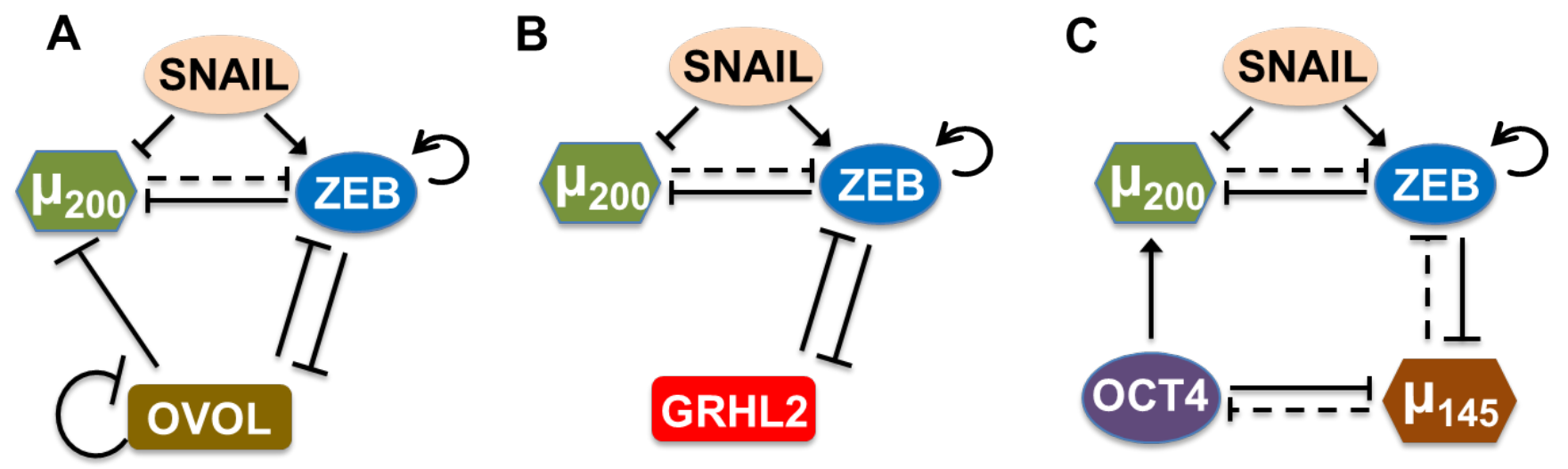

Figure 2: Coupling of core EMT circuit (miR-200/ZEB driven by SNAIL) with other 'phenotypic stability factors' (PSFs). A. OVOL forms a mutually inhibitory loop with ZEB, inhibits miR-200, and self-inhibits. B. GRHL2 forms a mutually inhibitory loop with ZEB. C. miR-145 and OCT4 mutually inhibit each other, miR-145 and ZEB also form a double negative loop, and OCT4 activates miR-200. Solid lines represent transcriptional regulation, while dotted lines represent miRNA-mediated regulation; arrows denote activation, bars for inhibition. Details of coupling for these PSFs with miR-200/ZEB are in SI section 1.

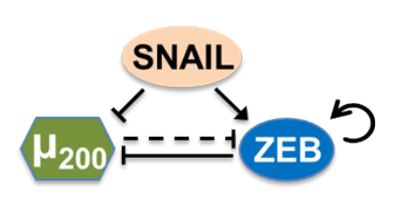

A

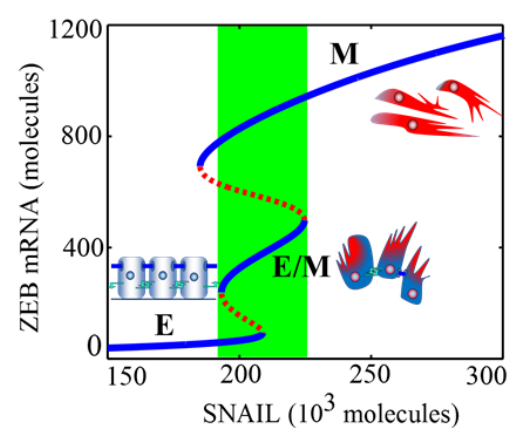

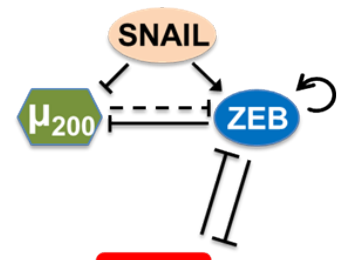

B GRHL2

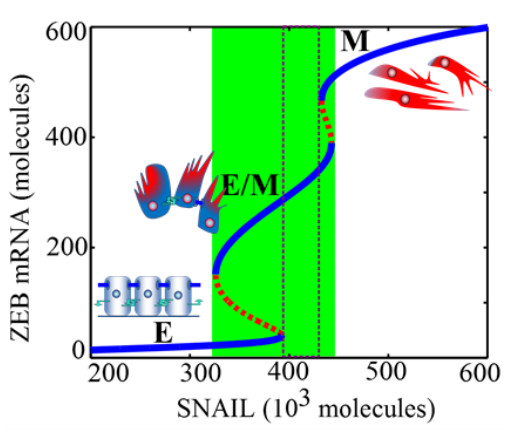

E

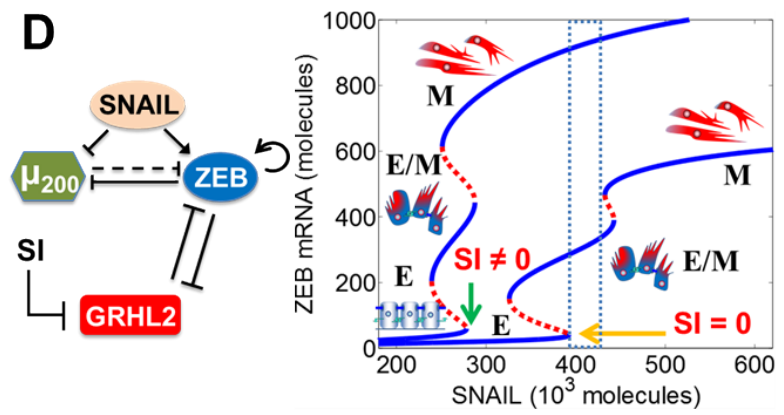

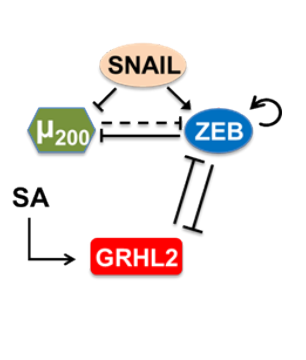
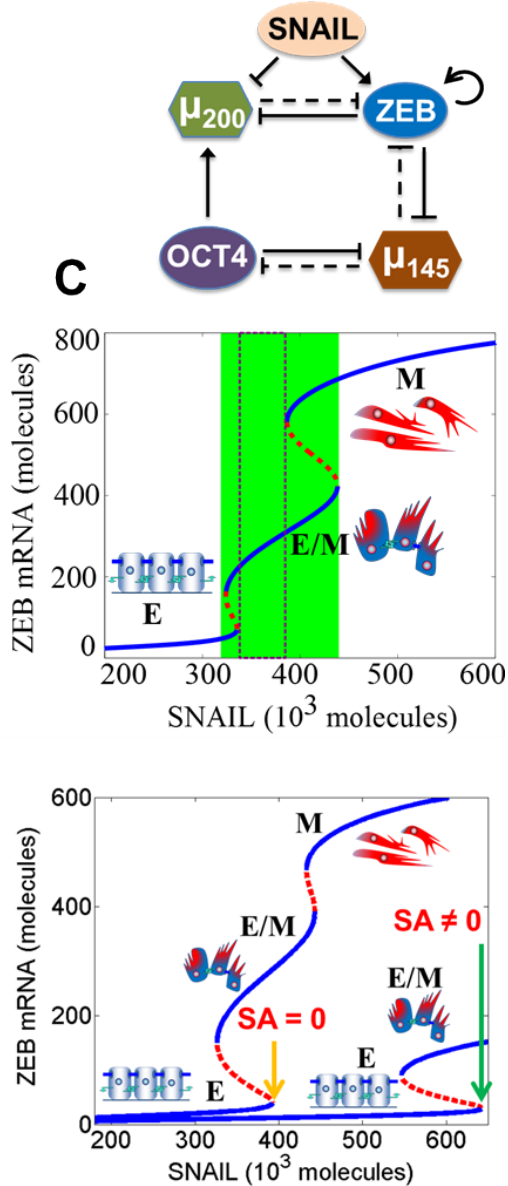

Figure 3: Dynamical system characteristics of miR-200/ZEB circuit when coupled with GRHL2 and miR-145/OCT4 separately. Bifurcation of mRNA levels of ZEB in response to SNAIL levels for A. miR-200/ZEB circuit, B. miR-200/ZEB/GRHL2 circuit, C. miR-200/ZEB/miR-145/OCT4 circuit, D. miR-200/ZEB/GRHL2 circuit when GRHL2 is inhibited by external signal SI, and E. miR-200/ZEB /GRHL2 circuit when GRHL2 is activated by external signal SA. Note the $\mathrm{x}$-axis range change from (A) to (B), (C), (D), and (E). The region marked by green in panels (A), (B), (C) represents the range of SNAIL levels for which the hybrid E/M phenotype can exist alone or as one of the multiple possible phenotypes; and that marked by dotted rectangle in (B), (C), and (D) represents the range of SNAIL levels for which the hybrid E/M phenotype can exist alone. Corresponding cartoons have been drawn alongside the phenotype. Blue solid lines denote stable steady states or phenotypes, and red dotted lines indicate unstable steady states. Yellow arrows in (D) and (E) indicate the SNAIL values at which cells can exit an epithelial phenotype for the control case (i.e. GRHL2 is not activated or repressed), whereas green arrows indicate the same for the case when GRHL2 is inhibited ((D)) or activated (E)). 
of GRHL2 and OVOL2 also restricted cell proliferation (Figure S6) - another cellular trait usually associated with EMT.

Next, we examined the levels of GRHL2 and OVOL (OVOL1/2) in two stable clonal cell lines derived from PC3 - PC3-Epi (epithelial clonal population), and PC3-EMT (mesenchymal clonal population) [30, 32], and observed significant differences in the ratio of $\mathrm{PC} 3-$ Epi/PC3-EMT levels for GRHL2 (142.12x), OVOL1 (174.43x), OVOL2 (25.89x), as well as ZEB1 (0.02x) and E-cadherin (50.43x) (Figure 4D, S7A), indicating that EMT or MET is associated with differential expression of these players. Overexpression of OVOL1 and OVOL2 in PC3-EMT cells can drive a MET [30]; but GRHL2 levels were not substantially upregulated (Figure 4D, S7A), indicating that GRHL2 and OVOL might be operating independent of each other in PC3 cells. However, the functional association between GRHL2 and OVOL might be tissue-specific, because GRHL2 can activate OVOL during nephric bud development [33] and trophoblast branching morphogenesis [34]. These contextual differences notwithstanding, GRHL2 and OVOL1/2 were recently demonstrated to be highly correlated with 'NCI60 consensus epithelial' (NEC) signature [35] - a list of genes such as CDH1 (E-cadherin) that are involved in maintaining adherens and/or tight junctions.
A
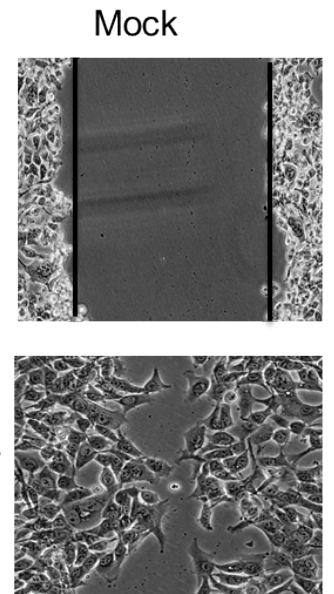

B
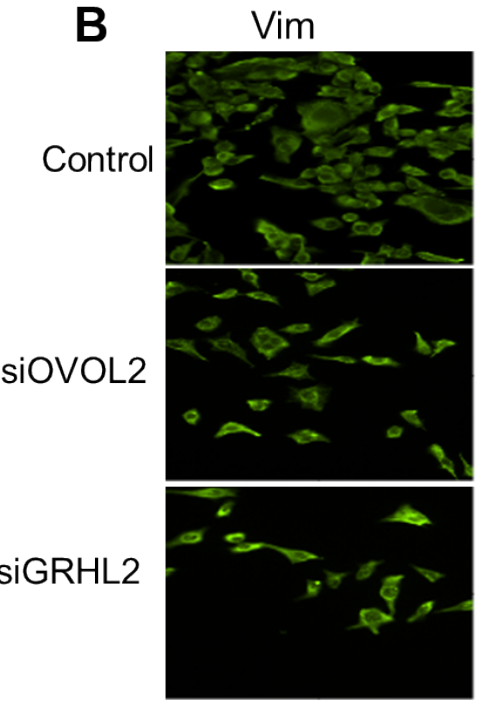

siGRHL2
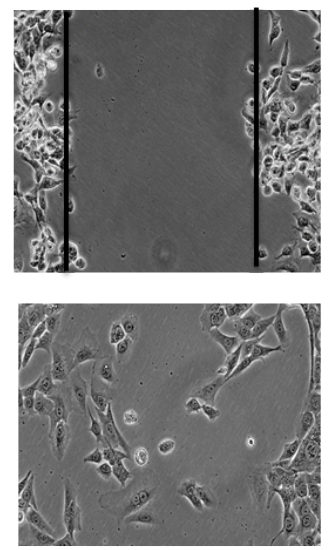

$\mathrm{CDH} 1$

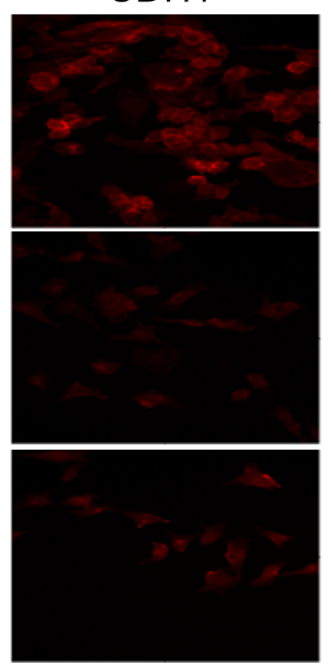

siOVOL2
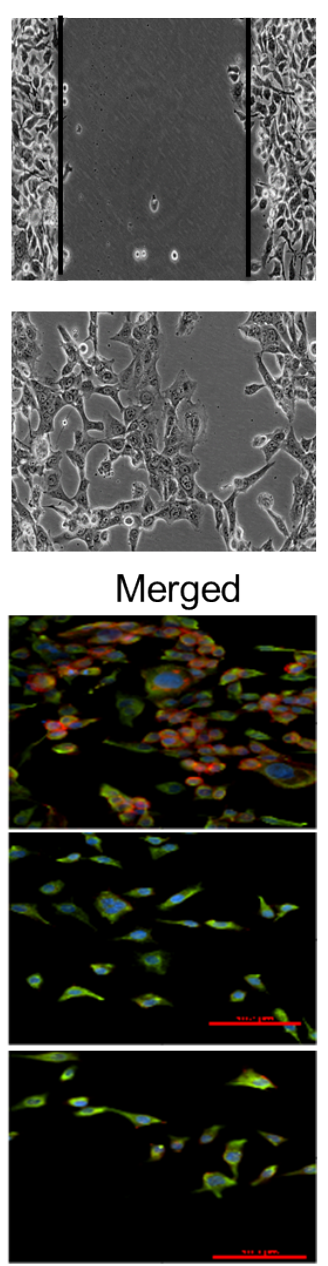

C
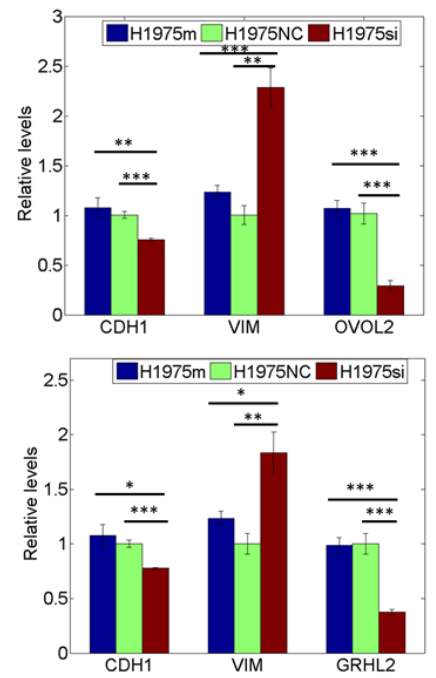

D

\begin{tabular}{c|c|c} 
Gene & Epi/EMT & EMT-OVOL2/EMT \\
\hline ZEB1 & 0.02 & 0.24 \\
CDH1 & 50.43 & 40.41 \\
GRHL2 & 142.12 & 5.37 \\
CDH3 & 16.02 & 17.79
\end{tabular}

\begin{tabular}{l|c|c} 
& $\mathbf{r}$ & P-value \\
\hline GRHL2 vs. OVOL2 & 0.7951 & $<0.0001$ \\
OVOL2 vs. CDH3 & 0.5025 & $<0.0001$ \\
GRHL2 vs. CDH3 & 0.4822 & $<0.0001$ \\
ZEB1 vs. GRHL2 & -0.6616 & $<0.0001$ \\
ZEB1 vs. OVOL2 & -0.6272 & $<0.0001$ \\
ZEB1 vs. CDH3 & -0.6052 & $<0.0001$ \\
\hline
\end{tabular}

Figure 4: Knockdown of GRHL2 and OVOL2 in H1975 cells, and expression values of GRHL2, CDH3, and OVOL in different PC-3 clones. A. Scratch assay of H1975 cells for the control case, and when treated with siRNA against GRHL2 and OVOL2, depicting different cell motility patterns. B. Expression of CDH1 (E-cadherin, red) and VIM (Vimentin, green) examined by immuno-fluorescence staining. Scale bar $100 \mu \mathrm{m}$. The blue color stains DAPI. C. Quantitative RT-PCR for CDH1, VIM, GRHL2, and OVOL2 after and before treatment with siRNAs against GRHL2 and OVOL2. Top panel is for siRNA against GRHL2, bottom one for that against OVOL2. H1975si shows the case when cells are treated with siRNA, H1975 NC denotes the negative control, and H1975m denotes mock case. ${ }^{*}, p<=0.05$; ${ }^{* *}, p<0.005$; and ${ }^{* * *}, p<0.0005$. Error bars represent standard deviation, $\mathrm{n}=3$. D. (top) Fold-change in expression levels ( $\log 2)$ of GRHL2, OVOL2, CDH3, and ZEB1 in PC3-Epi vs. PC3-EMT, and PC3-EMT-OVOL2 vs. PC-EMT clonal cell lines. (bottom) Correlation analysis for GRHL2, OVOL2, CDH3, and ZEB1 expression in NCI-60. Pearson correlation coefficients (r) and $p$-values (two-tailed) are given. 


\section{'Phenotypic stability factors' may predict poor prognosis}

Motivated by the correlation of GRHL2 and OVOL with the NEC signature, we investigated their levels in the NCI-60 cohort that has been classified as epithelial, mesenchymal, and hybrid $\mathrm{E} / \mathrm{M}$ groups of cell lines based on their CDH1/VIM expression ratio [36]. ZEB1 (the EMT-inducing TF in our core EMT circuit), OVOL2, and $\mathrm{CDH} 3$ (P-cadherin; a proposed marker of hybrid E/M phenotype [37]; 16.02x in PC3-Epi as compared to PC3-EMT) had statistically significant different levels in the different categories (E, M, and $\mathrm{E} / \mathrm{M})$ of the NCI-60 cell lines (Figure S7B). Further, the levels of GRHL2, OVOL2, and $\mathrm{CDH} 3$ were positively correlated with one another; and all of them negatively correlated with ZEB1 (Figure 4D). The strongest association observed was between GRHL2 and OVOL2, endorsing our model predictions about similar roles of OVOL and GRHL2 in mediating epithelial plasticity. In addition, OVOL1/2 is highly correlated with GRHL2 and CDH3 (positively) and with ZEB1 (negatively) in a panel of 877 cell lines [30] from The Cancer Cell Line Encyclopedia (CCLE) [38].

Finally, the levels of the PSFs OVOL2 and GRHL2, and the proposed marker for a hybrid $\mathrm{E} / \mathrm{M}$ phenotype $\mathrm{CDH} 3$ can correlate with poor overall survival (OS), metastasis -free survival (MFS), and relapse-free survival (RFS) across multiple tissue types (Figure 5, S8). Their low levels, indicative of a completely mesenchymal phenotype (Figure S3B, C), associate with a better survival, thereby arguing that complete EMT need not be the hallmark of aggressiveness. On the other hand, cells in breast and lung carcinomas usually shed some epithelial traits to migrate, invade and metastasize [2]. Therefore, the relatively high levels of CDH3, OVOL2, and GRHL2 in patients with poor survival might highlight the role of hybrid $\mathrm{E} / \mathrm{M}$ phenotype in metastatic progression, at least in these carcinomas. These survival curves are reminiscent of a recent study of 1678 independent human breast cancer samples indicating that a GRHL2-mediated gene-set pair can "effectively stratify patients showing significant differences in metastasis-free survival"[39], and are congruent with reports indicating that higher levels of GRHL2 correlate with shorter RFS in breast cancer patients [40], and with lower OS and RFS in colorectal cancer patients [41].

\section{Mathematical modeling predicts that GRHL2 can associate hybrid E/M phenotype with high tumor- initiation potential}

Consistent with the emerging idea of the role of hybrid E/M phenotype in metastatic progression [5], hybrid $\mathrm{E} / \mathrm{M}$ cells have also been recently shown to possess high tumor-initiating or stem-like properties, sometimes even higher than those possessed by cells with a complete EMT phenotype $[11,20]$. In other words, the 'stemness window' (range of conditions under which cells attain stem-like traits) lies somewhere mid-way on an 'EMT axis' (with $\mathrm{E}$ and $\mathrm{M}$ as its two ends) [18, 42] as postulated earlier [43]. Here, we investigate how PSFs such as GRHL2 affect the positioning of the 'stemness window' on the 'EMT axis'.

The EMT-stemness interplay is regulated by coupling between the core EMT module (miR-200/ZEB) $[25,29]$ and the core stemness module (LIN28/let-7) [44-47]. They couple to each other via two links - miR200 inhibits LIN28 [48] (hereafter called 'feed-forward coupling') and let-7 inhibits ZEB via HMGA2 [49, 50] (hereafter called 'feed-backward coupling') (Figure 6A, 6B). The strengths of these links are represented by $\alpha 1$ and $\alpha 2$ respectively. Both $\alpha 1$ and $\alpha 2$ are defined to be between 0 and 1 ; the larger their values, the stronger the corresponding inhibition. It may be noted that we are ignoring the feedback from OCT4 to miR-200 in our current analysis. Previous experimental and theoretical analysis has indicated that cells are most likely to be stemlike at intermediate levels of OCT4 (a downstream target of LIN28); both very high and very low levels of OCT4 cause the cells to differentiate (51-55). Taking this clue, we choose a 'stemness window', i.e. the representative range of levels of OCT4 for which the cells have the maximum likelihood to gain stemness to be $(0.25-0.65)$ relative to the saturation level of OCT4 when it is activated by the maximum levels of LIN28 (marked by the red shaded region in Figure 6E, 6F). Notably, this range of OCT4 levels chosen for the 'stemness window' is likely to be context-specific; the results shown here are for a specific range to illustrate the concept.

To investigate the effect of GRHL2 on the EMTstemness interplay, we calculate the steady states of the miR-200/ZEB/LIN28/let-7 and miR-200/ZEB/LIN28/let7/GRHL2 circuits - both driven by SNAIL - at different values of $(\alpha 1, \alpha 2)$, and plot the 'stemness region', i.e. range of $(\alpha 1, \alpha 2)$ values for which these phenotypes - E, $\mathrm{E} / \mathrm{M}$, and $\mathrm{M}$ - can gain stemness as measured by levels of OCT4. We start with high levels of SNAIL such that at no coupling $(\alpha 1=\alpha 2=0)$, all cells are in either a mesenchymal or hybrid $\mathrm{E} / \mathrm{M}$ phenotype and possess stem-like traits (phase $\{E / M, M\}$ at $\alpha 1=\alpha 2=0$ in Figure $6 \mathrm{~A})$. In the absence of GRHL2, a large range of values of $(\alpha 1, \alpha 2)$ allow any of the three phenotypes (E, M, and $\mathrm{E} / \mathrm{M})$ to gain stemness, thereby reflecting an enriched flexibility of the 'stemness window' on the 'EMT axis'. However, upon including GRHL2, this tristable phase $\{\mathrm{E}$, $\mathrm{E} / \mathrm{M}, \mathrm{M}\}$ disappears (compare the black area in Figure $6 \mathrm{D} v s$. the absence of that in Figure 6C). Furthermore, the miR-200/ZEB/LIN28/let-7/GRHL2 circuit enables, for a significant range of the values of $(\alpha 1, \alpha 2)$, an exclusive association of the hybrid E/M phenotype with stemness, an association not observed in the miR-200/ZEB/LIN28/ 

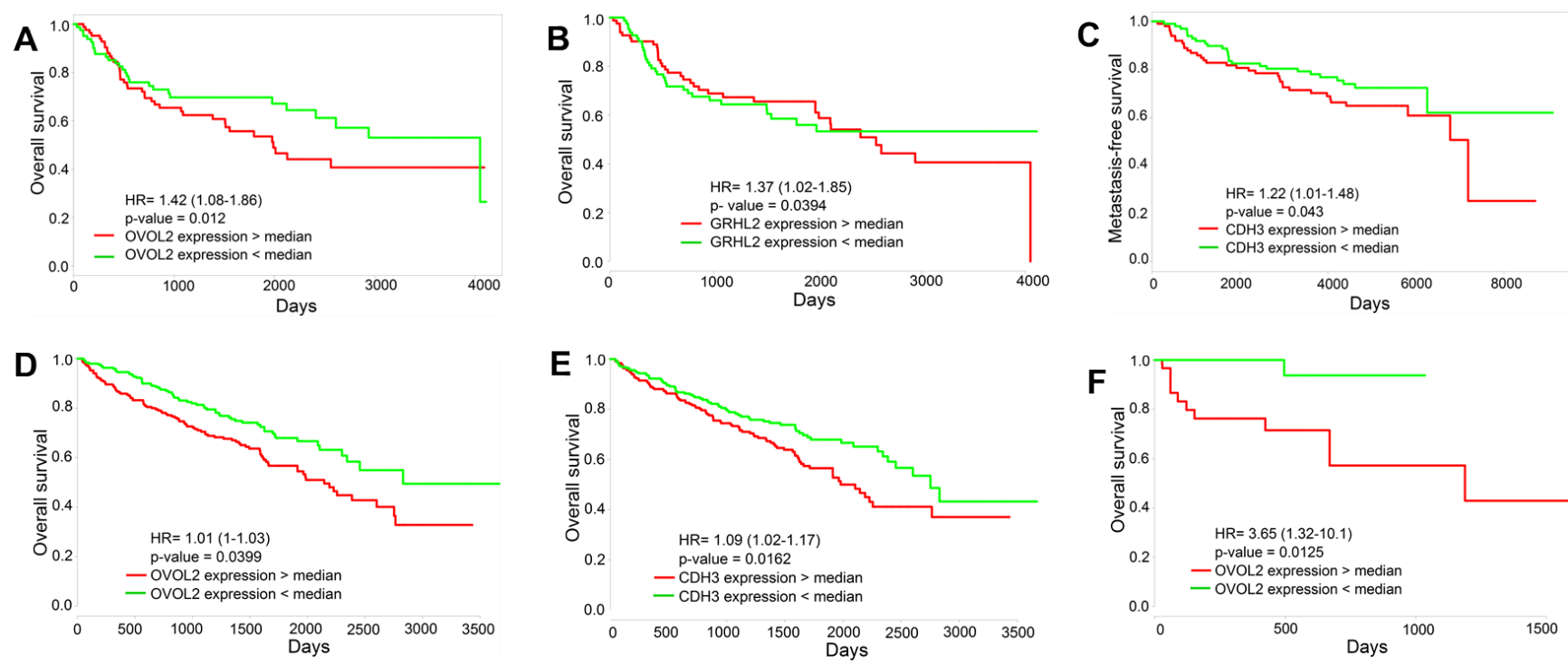

Figure 5: Survival analysis. Overall survival and metastasis-free survival for the expression of GRHL2, OVOL2 and CDH3 individually in multiple tissue types - A., B. GSE13507 $(n=164)$, C. GSE7390 $(n=197)$, D., E. TCGA_KIRC $(n=505)$, F.TCGA_COAD $(n=120)$.

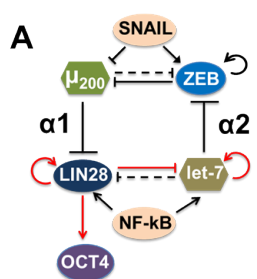

C

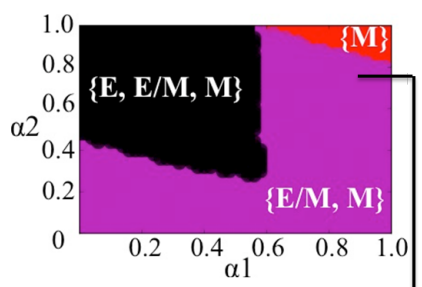

E
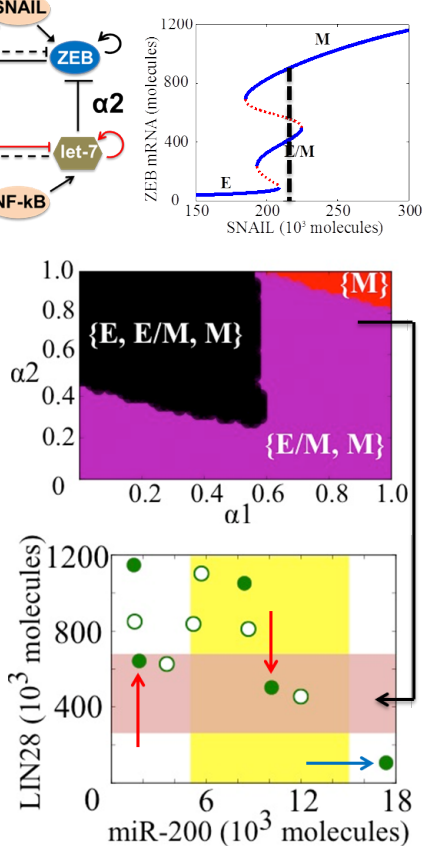

B

B
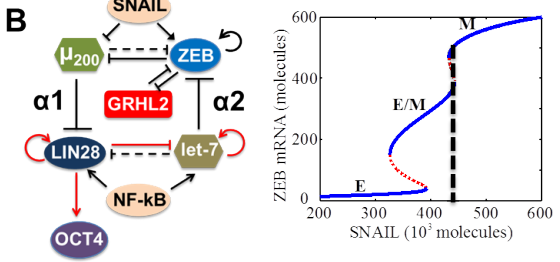

D

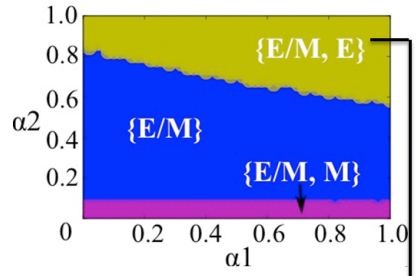

F

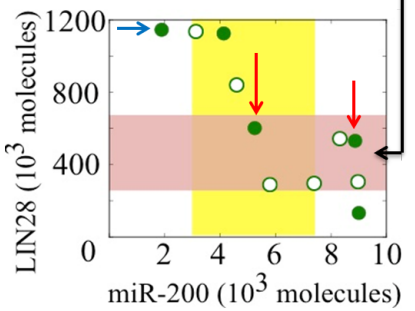

Figure 6: State-space characteristics of coupled networks miR-200/ZEB/LIN28/let-7 and miR-200/ZEB/LIN28/let-7/ GRHL2, when cells are in $\{\mathbf{E} / \mathbf{M}, \mathbf{M}\}$ phase at $\boldsymbol{\alpha} \mathbf{1}=\boldsymbol{\alpha} \mathbf{2}=\mathbf{0}$. A., B. miR-200/ZEB /LIN28/let-7 and miR-200/ZEB/GRHL2/LIN28/ let-7 circuits respectively. Black solid lines represent transcriptional regulation; red lines denote translational self-activation of LIN28 (46) and activation of its own processing by the microRNA let-7 (47), and dotted lines denote miRNA-mediated regulation. The parameters $\alpha 1$ and $\alpha 2$ denote the strength of the 'feed-forward coupling' (miR-200 inhibiting LIN28) and 'feed-backward coupling' (let-7 inhibiting ZEB) respectively, and lie between 0 and 1. Larger values denote stronger inhibition. The dashed line in the bifurcation diagrams next to the circuits shows the phase in which cells are present when there is no coupling between EMT and stemness circuits $(\alpha 1=\alpha 2=0)$. Steady state diagram and the phase diagram in every column are for the circuit drawn in the topmost row of that column. C. Phase diagram of the circuit miR-200/ZEB/LIN28/let-7 representing the values of $(\alpha 1, \alpha 2)$ for which the different phenotypes can lie in the stemness window, for SNAIL $=220 * 10^{3}$ molecules and NF-kB $=25^{*} 10^{3}$ molecules. E. Phenotypic map of the coupled circuit at $\alpha 1=\alpha 2=0.8$ and at driving signals SNAIL $=220^{*} 10^{3}$ molecules and NF-kB $=25^{*} 10^{3}$ molecules. Red shaded area shows the 'stemness window' based on relative OCT4 levels, and yellow shaded area represents the range of miR-200 levels for the existence of the hybrid E/M phenotype, as noted in (29) for (miR-200/ZEB) circuit and in SI section 10 for (miR-200/ZEB/GRHL2) circuit. D., F. represent a similar case for (C), (E) respectively but for the circuit with GRHL2, therefore SNAIL $=440^{*} 10^{3}$ molecules. Different colors represent different combinations of phenotypes that can gain stemness. The red arrows highlight the phenotypes that lie in the 'stemness window', and blue arrows denote some cases where the phenotypes lie outside of the 'stemness window'. Green filled circles denote the stable steady states, and green hollow circles show the unstable steady states of the coupled circuits as denoted in (A) and (B). 
let-7 circuit (compare the blue area in Figure 6D vs. the absence of that in Figure 6C). Also, at strong bidirectional coupling $(\alpha 1=\alpha 2=0.8)$, in the absence of GRHL2, both $\mathrm{E} / \mathrm{M}$ and $\mathrm{M}$ phenotypes can attain stemness (cells can also attain an $\mathrm{E}$ phenotype, but E phenotype lies outside the 'stemness window') as shown by blue arrow in (Figure $6 \mathrm{E})$, whereas in presence of GRHL2, both $\mathrm{E}$ and E/M phenotypes can gain stemness but the $\mathrm{M}$ phenotype is precluded (compare the green region in Figure 6D vs. the absence of that in Figure 6C; and the states lying in 'stemness window' in Figure 6F vs. those in Figure 6E). The cells can still attain $\mathrm{M}$ phenotype, but it lies outside the 'stemness window' (see blue arrow in Figure 6F). The stem-like features of both $\mathrm{E}$ and $\mathrm{E} / \mathrm{M}$ phenotypes are reminiscent of experiments showing two sub-populations of Cancer Stem Cells (CSCs) - one more E-like, and the other commensurate with a hybrid E/M phenotype [56].

Next, we choose a different value of SNAIL such that at $\alpha 1=\alpha 2=0$, all cells are mesenchymal ( $\{\mathrm{M}\}$ phase) and can gain stemness. In the absence of GRHL2, only
A
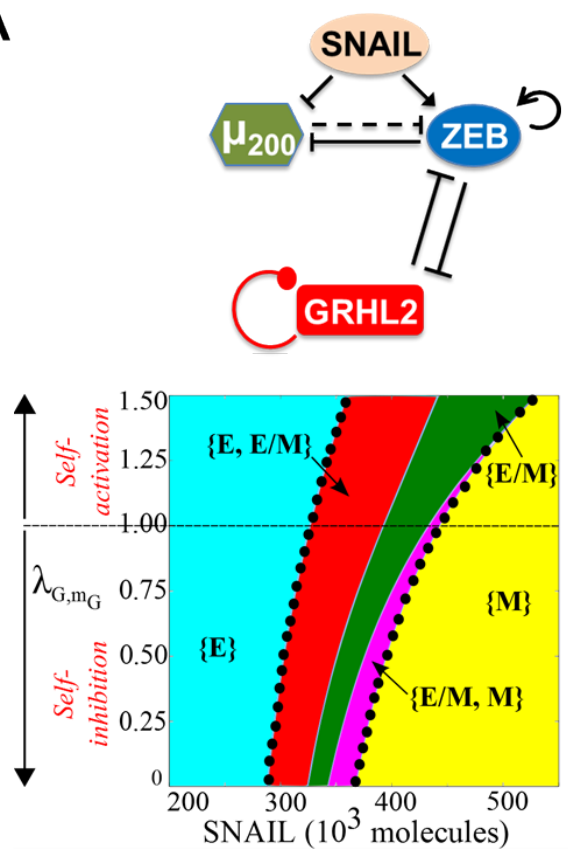
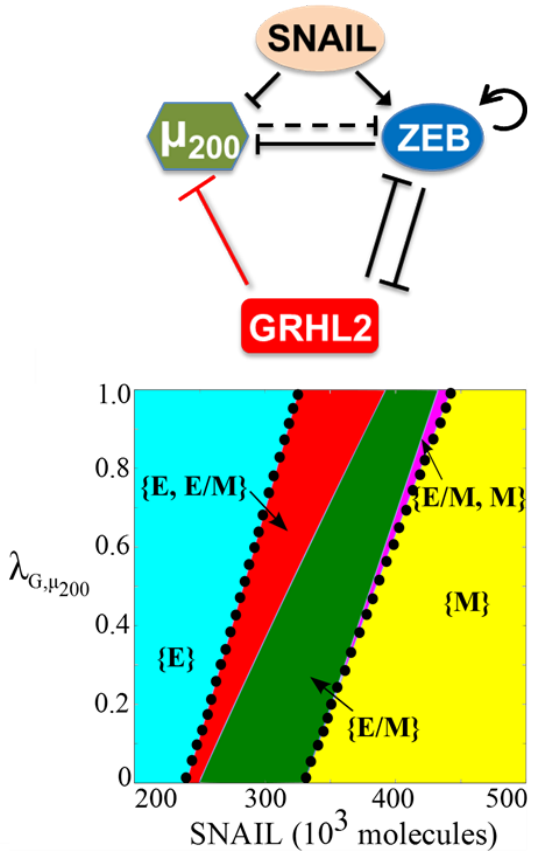

SNAIL

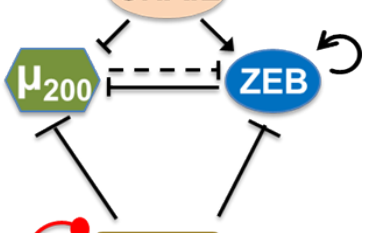

OVOL

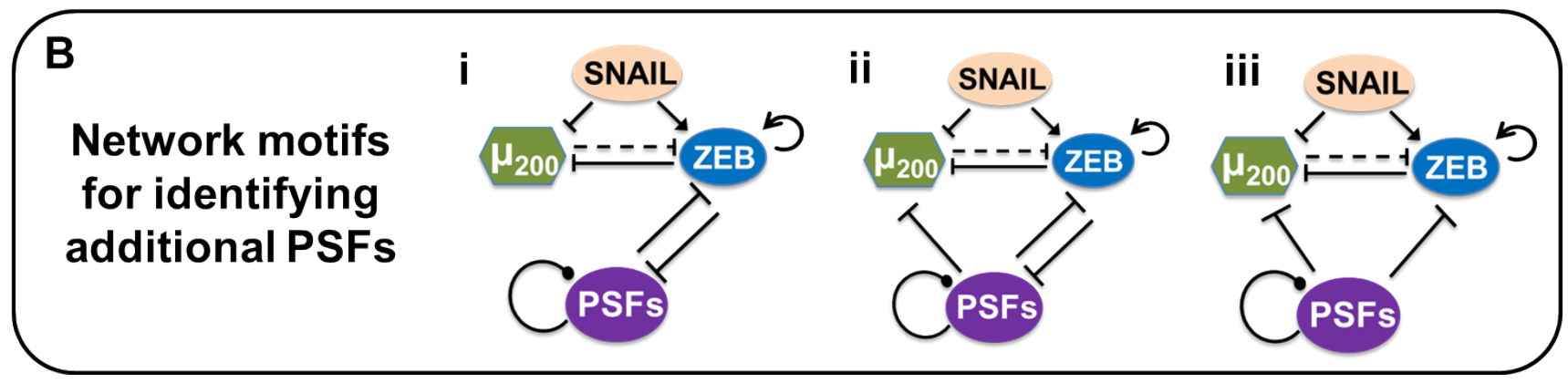

Figure 7: Network motifs that help maintain the hybrid E/M phenotype. A. Top row denotes the different circuits that are investigated; the link whose strength is varied is depicted in red. Bottom row shows the phase-diagram of the miR-200/ZEB/GRHL2 circuit when driven by SNAIL and including a hypothetical self-regulatory link for GRHL $\left(\lambda_{G, m_{G}}\right.$ denotes the strength of the self-regulation;

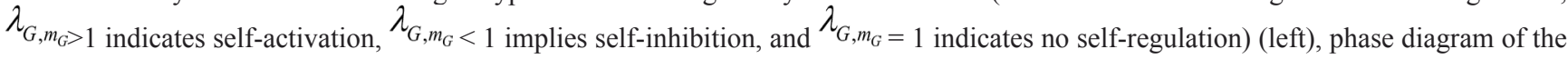
miR-200/ZEB/GRHL2 circuit when driven by SNAIL and a hypothetical inhibitory link from GRHL2 to miR-200 (the smaller the value

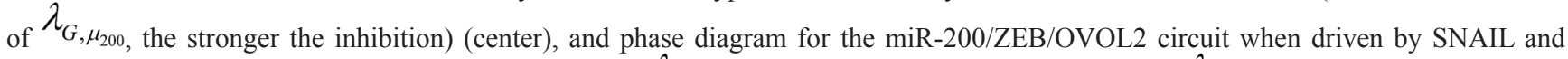

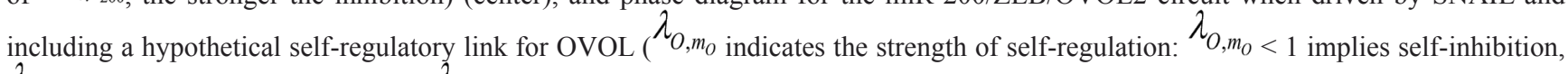
$\lambda_{O, m_{O}>1}$ implies self-activation, and $\lambda_{O, m_{O}}=1$ shows no self-regulation) (right). In all these phase diagrams, the black dots bound the area of all phases that contain $\mathrm{E} / \mathrm{M}$ as one of its phenotypes, and dark green region shows the phase when hybrid E/M phenotype need not co-exist with other phenotypes - $\{\mathrm{E} / \mathrm{M}\}$ B. Proposed network motifs for identifying PSFs. The dot at the end of the self-regulatory link indicates that the PSF can potentially either self-activate or self-inhibit. 
the $\mathrm{M}$ phenotype can gain stemness for almost the entire range of $(\alpha 1, \alpha 2)$ values; however, including GRHL2 enables a large range of the values of $(\alpha 1, \alpha 2)$ in which only the hybrid E/M phenotype lies in the 'stemness window' (Figures S9,10). Therefore, collectively, these results suggest GRHL2 excludes the association of mesenchymal $(\mathrm{M})$ phenotype with stemness, and promotes the association of hybrid E/M phenotype with stem-like traits. In other words, GRHL2 maintains the stemness window closer to the hybrid E/M phenotype (mid-point of 'EMT axis'). Collectively, these results illustrate that GRHL2 can not only stabilize the hybrid E/M phenotype, but also enhances the likelihood of the hybrid E/M cells to be 'stem-like' or gain stemness.

Although yet to be directly experimentally tested, these predictions are congruent with recent reports demonstrating that overexpression of GRHL2 can promote tumor growth and metastasis in vivo [40, 57], and offer a possible explanation for downregulation of GRHL2 suppressing tumor growth in vivo [57].

\section{Proposing several network motifs for identifying 'phenotypic stability factors'}

Intrigued by the similar role played by OVOL, GRHL2, and miR-145 in stabilizing the hybrid E/M phenotype, we analyzed the similarities and differences in these circuits - (miR-200/ZEB/OVOL), (miR-200/ ZEB/GRHL2), and (miR-200/ZEB/miR-145/OCT4). For ease of comparison, the (miR-200/ZEB/ miR-145/ OCT4) circuit is effectively reduced to a (miR-200/ZEB/ miR-145) circuit such that similar to OVOL and GRHL2, miR-145 inhibits ZEB and is inhibited by ZEB relatively weakly $(26,30,58-60)$ as compared to the inhibition of miR-200 by ZEB(25) (Figure S11).

These three circuits have two key differences (a) OVOL self-inhibits, miR-145 self-activates (in the effective circuit), and GRHL2 has no self-regulation (although it can self-activate in some biological contexts $[27,61]$ ); and (b) OVOL and miR-145 (in the effective circuit) inhibit miR-200, but GRHL2 does not. Therefore, we investigate how two hypothetical links - self-regulation of GRHL2 and inhibition of miR-200 by GRHL2 affect the range of parameters for the existence of $\mathrm{E} / \mathrm{M}$ phenotype. We observe that the total area corresponding to the phases that contain E/M as one of the states or the only state increases monotonically as the strength of self-inhibition of GRHL2 is decreased and/or when the strength of self-activation of GRHL2 is increased (the area bounded by black dots in Figure 7A, left panel).

The other link - inhibition of miR-200 by GRHL2 - is represented by $\lambda_{G, \mu_{200}}(<1)$. We observe that the total area corresponding to phases containing E/M as one of the states does not change significantly by including an inhibitory link to miR-200 (the area bound by black dots in
Figure 7A, middle panel). Therefore, neither the inhibitory link to miR-200 nor the self-regulatory action of the 'phenotypic stability factor' (PSF) qualitatively changes their behavior of stabilizing the hybrid E/M phenotype. Based on these results, we propose two network motifs to identify PSFs; if a particular transcription factor or miRNA couples with (miR-200/ZEB) in one of these two topologies, it is likely to act as a PSF for the hybrid E/M phenotype (Figure 7B, i-ii). In these motifs, a PSF forms a mutually inhibitory loop with ZEB, may inhibit miR-200, and may self-activate or self-inhibit (as shown by the dot at the end of the self-regulatory link) (Figure 7B, i-ii).

We also propose another network motif for identifying PSFs - a molecular player that can inhibit both miR-200 and ZEB (Figure 7B, iii). This motif is proposed by introducing the miR-200/ZEB/OVOL circuit where the inhibitory feedback from ZEB to OVOL is absent - as observed during mammary morphogenesis and epidermal development [14, 62] (Figure 7A, right panel). Again, relieving the self-inhibition and/or increasing the self-activation of OVOL only increases the total area corresponding to the phases that contain E/M as one of the phenotypes (the area bound by black dots in Figure 7A, right panel). Such an 'incoherent' external inhibitory signal that inhibits both miR-200 and ZEB can also increase the association of hybrid E/M phenotype with stemness (Figure S12-S14). Collectively, these three motifs can be utilized to identify additional factors that can stabilize a partial EMT.

Notably, the factors that form a mutually inhibitory loop with miR-200 instead of that with ZEB (Figure 7B, i-ii) cannot stabilize the hybrid E/M phenotype (Figure S15). Similar results are observed (Figure S15) when both miR-200 and ZEB are being activated instead of being inhibited (Figure 7B, iii), thereby illustrating the specificity of the proposed network motifs that can maintain a hybrid E/M phenotype. A different recent modeling attempt identified network motifs that can maintain a completely mesenchymal (M) or epithelial (E) phenotype $[63,64]$, but ours, to the best of our knowledge, is the first attempt to identify motifs that can stabilize a hybrid E/M or partial EMT phenotype.

\section{DISCUSSION}

EMT and MET are fundamental processes during development, homeostasis, and diseases such as cancer and fibrosis that enable reversible bidirectional transitions between epithelial (E) and mesenchymal (M) phenotypes [2]. They have been largely considered to be binary or 'all-or-none' processes [1], but recently, a partial EMT or hybrid E/M phenotype has been increasingly recognized. This recognition is even more recent in EMT associated with cancer [17, 19, 22, 65-69] as compared to EMT associated with embryonic development and wound repair/tissue regeneration $[4,12,70,71]$. This phenotype 
has been assumed to be a 'metastable' or transient state $[12,13]$ and CTCs have been observed to alter their EMT status dynamically [22], but recent experiments suggest a hybrid E/M phenotype can be stable [14, 72, 73]. Whether it is truly 'metastable' from a dynamical systems perspective [74] has been elusive.

The results here indicate that partial EMT need not be a 'metastable' transitory state attained en route to EMT, rather it can reflect a stable phenotype, especially in the presence of 'phenotypic stability factors' (PSFs) such as OVOL2, GRHL2, and miR-145. Experiments - including those shown here - showing that OVOL and GRHL2 knockdown can impair collective cell migration - the hallmark of partial EMT - and induce a complete EMT suggest that these PSFs can act as a "critical molecular brake on [a complete] EMT"[14]. Furthermore, GRHL2 can activate OVOL during nephric bud development and both of them induce the expression of epithelial effectors such as CDH1 [33], thereby forming a coherent feedforward loop (FFL) [75], a common network motif that acts as a "stabilizer of target gene expression"[33]. The arrangement of two PSFs GRHL2 and OVOL in a FFL further strengthens the emerging notion that the hybrid E/M phenotype "defines [a] normal cell identity" and is not "necessarily [an] unstable transitory state"[76].

OVOL [18] and GRHL2 can not only stabilize a hybrid E/M phenotype, but also have been predicted to associate hybrid E/M phenotype with stemness, thereby suggesting a common design principle - stabilizing the hybrid E/M phenotype also potentially increases its likelihood to gain stemness While the role of these PSFs in maintaining the 'stemness window' around the midpoint of the 'EMT axis' [18, 42] remains to be directly experimentally tested, the functional association of a hybrid E/M phenotype with stemness has been reported during EMT in developmental (type I EMT) [77], regenerative (type II EMT) [78], as well as pathological contexts (type III EMT) [79, 80]. Specifically, recent in vitro analysis of HMLER cells indicate that hybrid E/M cells form ten times more mammospheres as compared to a similar number of $\mathrm{E}$ and $\mathrm{M}$ cells [11]. Similar behavior of hybrid E/M cells was observed in multiple lung cancer cell lines [20]. Further, in ovarian and breast cancer metastasis, in vivo tumor growth is largely driven by hybrid E/M cells [81, 82]. Consistently, a transient activation, but not necessarily a prolonged activation, of EMT-inducing transcription factors including TWIST and SNAIL can significantly increase the tumor-initiating potential $[83,84]$; suggesting that partial EMT is more stem-like than complete EMT.

The association of hybrid E/M phenotype with stemness [11, 45] is also strengthened by experiments indicating a link between the hybrid E/M phenotype and both de novo and acquired drug resistance [5]. Furthermore, the chemo-tolerant subpopulation in both basal-like and luminal breast cancer cell lines co-express both an epithelial marker CD24 and a mesenchymal marker CD44 [85], indicating its hybrid E/M status [11]. These CD24+ CD44+ cells also form more aggressive tumors in vivo as compared to the CD24-CD44+ (M) population [85]. In addition, a recent study demonstrated that co-treating the cells with EMT-inducing TGF $\beta$ and EMT-inhibiting Retinoic Acid (RA) [86] can enrich the hybrid E/M subpopulation (CD24+ CD44+) that is highly drug resistant [87].

Recent experiments indicate another possible PSF - $\Delta \mathrm{Np} 63 \alpha$ - that can induce a partial EMT in basal-like breast cancer cells by activating Slug (SNAIL2) as well as inhibiting ZEB via miR-205 [76]. Notably, CDH3 (P-cadherin) - a proposed marker of the hybrid E/M phenotype - is a downstream target of $\Delta \mathrm{Np} 63 \alpha$ [37]. The expression of $\Delta \mathrm{Np} 63 \alpha$, Slug, and P-cadherin (CDH3) in myoepithelial cells $[37,76]$, and the activation of the p63 gene by GRHL2 in keratinocytes [61], further argues for the role of $\Delta \mathrm{Np} 63 \alpha$ in inducing a partial EMT. Whether $\Delta \mathrm{Np} 63 \alpha$ truly behaves as a PSF requires more careful analysis from a modeling perspective. For instance, the current model lumps together Snail1 and Snail2 (Slug) as SNAIL family, but they should be considered as two distinct entities to appreciate possible contextual differences between Snaill and Slug - $\Delta \mathrm{Np} 63 \alpha$ activates Slug [76] but not necessarily SNAIL1, and Slug and Snaill can have different roles in developmental and oncogenic EMT and may even inhibit each other [88, 89]. Not all PSFs need be expressed or be functionally active in the same context; also, their coupling with miR-200/ZEB might vary slightly in different tissues [90]. Therefore whether they act redundantly or synergistically is likely to be tissue-specific. Besides, the players that maintain a hybrid E/M state in fibrosis [91, 92] might be different from those reported here.

We also show two lung cancer cell lines classified as hybrid E/M based on population-based measurements - one of which predominantly contains hybrid E/M cells (H1975), and the other is largely an admixture of E and M cells (H2291). Importantly, H1975 cells can maintain their hybrid E/M phenotype for over two months in culture, and display collective migration, indicating their stable phenotype. Similar hybrid E/M cells co-expressing E and $\mathrm{M}$ markers have recently been reported in breast and lung cancer cell lines $[11,17,20]$, yet most previous reports on partial EMT and those indicating EMT to be a spectrum of phenotypes are largely at an ensemble level [19, 64$66,93,94]$, thereby being inconclusive whether they contain admixtures of $\mathrm{E}$ and $\mathrm{M}$ cells, or individual hybrid $\mathrm{E} / \mathrm{M}$ cells. Of course, these two manifestations of hybrid $\mathrm{E} / \mathrm{M}$ - population level and single-cell level - need not be mutually exclusive, for instance, HMLER cells contain subpopulations for E, M and hybrid E/M cells [11].

How many intermediate states exist en route EMT, what are their different gene expression profiles, and what is their relative stability? These challenging 
questions remain unanswered, providing a fertile ground for integrating modeling and experimental approaches $[17,63,64,95,96]$. The direct coupling of the PSFs OVOL and GRHL2 to ZEB instead of SNAIL strengthens the claim that (miR-200/ZEB) is the "motor of cellular plasticity' that shepherds epithelial-hybrid-mesenchymal transitions [29], and might be in slight disagreement with 'equal' decision-making potential of (miR-200/ ZEB) and (miR-34/SNAIL) loops [95]. However, a detailed discussion on how specific the predictions are to the modeling framework adopted here vis-à-vis other modeling frameworks $[17,95]$ is outside the scope of this article, and is being dealt in sufficient detail, including experimental data, elsewhere (Jia et al. in preparation). Besides, a recent integrative study indicates that OVOL can mediate two intermediate states between $\mathrm{E}$ and M phenotypes - a hybrid E/M, and a 'naïve' one [17]. Conceptually, this observation is congruent with our proposition that OVOL expands the range of parameters (i.e. physiological conditions) for the existence of a hybrid E/M phenotype [16]. Moreover, the stable existence and functional significance of the proposed 'naïve' state [17] remains to be shown experimentally. Further accumulation of experimental evidence for intermediate state(s) is also expected to better integrate 'bottom-up' and 'topdown' modeling approaches that have been adopted to characterize the signature of hybrid E/M phenotype the former considers a few 'core' components identified experimentally $[97,98]$ and focuses on elucidating their specific functions and the emergent outcome of their quantitatively-characterized interactions [17, 29, 95, 96], while the latter considers a much larger network and attempts to lay out all different possible steady states of the network $[63,99]$.

Overall, using a network-biology approach, we present three 'phenotypic stability factors' (PSFs) OVOL, GRHL2, and miR-145 - that can maintain a hybrid $\mathrm{E} / \mathrm{M}$ phenotype and have been predicted to increase the likelihood of hybrid E/M phenotype in gaining stemness. This proposed dual role of PSFs can be crucial during cancer metastasis as they can both enable collective migration of tumor cell clusters and confer these clusters with high tumor-initiating properties. The proposed contribution of PSFs to metastatic load is supported by lower metastasis-free survival, relapse-free survival, and overall survival time for patient samples overexpressing one or more of GRHL2, OVOL2, and CDH3. Targeting these PSFs therapeutically can help break the migrating CTC clusters that act as the primary 'bad actors' of metastasis because of the multiple advantages of cluster migration - resistance to anoikis, more tumor-initiating potential, ease of intravasation and extravasation, and finally the 'priming' for subsequent metastatic dissemination [5]. These results suggest a rethinking in the diagnostic strategy. Recent attempts have largely focused on isolating single CTCs [7], however, isolating and characterizing CTC clusters [100] might be the most effective and much-needed diagnostic approach [5].

Notably, the identification of PSFs presented here is by no means comprehensive. These examples studied here allow us to define a set of network motifs that will allow us to search for other PSFs. Specifically, we propose three particular network topologies that can be used to mine for other similar 'phenotypic stability factors' (PSFs) - (a) a double negative feedback loop with ZEB, (b) inhibition on both miR-200 and ZEB, and (c) a double negative feedback loop with ZEB as well as inhibiting miR-200. In all these three cases, the PSF can self-regulate positively or negatively. With a surging interest in mapping and modeling the signaling pathways regulating metastasis $[45,63,101-104]$, the theoretical approach presented here can serve as a template to elucidate the effect of many intracellular and extracellular signals in regulating EMT dynamics and governing the relative stability of the E, M and $\mathrm{E} / \mathrm{M}$ phenotypes.

\section{MATERIALS AND METHODS}

\section{Cell line and siRNA transfection}

The H1975 cell line was authenticated and free from mycoplasma, was grown in RPMI 1640 with $10 \%$ FBS and $1 \%$ penicillin/streptomycin. siRNA against GRHL2, OVOL2 and scrambled control siRNA were purchased from Sigma (Hs01_00105962, Hs01_00105964, Hs02_00357526, Hs01_00357580). The siRNA transfection was performed with Lipofectamine 2000 (catalog\# 11668-019; Invitrogen), according to the manufacturer's instructions. An siRNA concentration of $50 \mathrm{nmol}$ was used for a $35 \mathrm{~mm}$ dish of H1975 cells.

\section{Mathematical modeling of EMT signaling network and coupled EMT-stemness network}

We mathematically modelled the coupling of the core EMT network (miR-200/ZEB) with GRHL2 and miR-145 (SI sections 1, 2) by generalizing and extending our previous theoretical framework [29]. We also coupled the core EMT and stemness modules (miR-200/ZEB and LIN28/let-7 respectively) along with GRHL2 (SI sections 1,2 ) by extending our previous theoretical framework for EMT-stemness coupling [18, 45]. The parameters involved (SI Tables 1-3) in the model have been obtained from the literature or estimated from analyzing experimental data. The sensitivity analysis of the model (Figures S1,-S2) indicates its robustness to parameter change. The number of molecules of miRNAs, mRNAs, and proteins has been estimated based on their typically observed concentrations in eukaryotic cells. For instance, the concentration of a protein is $10 \mathrm{nM}-1 \mu \mathrm{M}$ [105] which when multiplied with 
typical volume of a mammalian cell (100-1000 um³), amounts to around 6 million molecules, commensurate with the range depicted in the simulations in this paper. Similarly, the ratio of protein/mRNA of a particular gene can be $\sim 3000$ [106], therefore the number of mRNA molecules of a gene can be estimated to be of the order of a thousand. In addition, the number of microRNA molecules in a cell is approximately10000 [107].

\section{RT-PCR analysis and immunofluorescence}

Complementary DNA samples were prepared using a High-Capacity cDNA Reverse Transcription Kit (Life Technologies). A TaqMan PCR assay was performed with a 7500 Fast Real-Time PCR System using TaqMan PCR master mix, commercially available primers, FAM ${ }^{\mathrm{TM}}$ labeled probes for GRHL2, OVOL2, CDH1, Vimentin and VICTM-labeled probes for $18 \mathrm{~S}$, according to the manufacturer's instructions (Life Technologies). Each sample was run in triplicate. Ct values for each gene were calculated and normalized to $\mathrm{Ct}$ values for $18 \mathrm{~S}(\Delta \mathrm{Ct})$. The $\Delta \Delta \mathrm{Ct}$ values were then calculated by normalization to the $\Delta \mathrm{Ct}$ value for control.

For immunofluorescence, cells were fixed in $4 \%$ paraformaldehyde, permeabilized in $0.2 \%$ Triton X-100, then stained with anti-CDH1 (1:100; Abcam) and antivimentin (1:100; Cell Signaling Technology). The primary antibodies were then detected with Alexa conjugated secondary antibodies (Life technologies). Nuclei were visualized by co-staining with DAPI.

\section{Wound-healing assay}

A scratch or wound-healing assay was performed to determine cell migration using confluent cultures (80\%-90\% confluence). Briefly, cells $\left(1 \times 10^{5}\right.$ cells $\left./ \mathrm{ml}\right)$ were seeded in 6-well tissue culture plate and grown to confluence. Cells were starved for 24 hours using $0.2 \%$ serum in growth media and treated with mitomycin to minimize cell proliferation. The next day, the confluent monolayer was scratched with a sterile p200 pipet tip and media replaced with complete growth media. Images were acquired at 0 and 12 hours; the assay was performed at least twice per cell line. The quantification of area covered was done by ImageJ software.

\section{Kaplan-Meier plot analysis}

Kaplan-Meier plots were generated using the online tool ProgGene [108]. The patients were classified into high or low based on the median level of expression for a given gene.

\section{Cell viability assay}

Cells were seeded at 5000 cells/well in 96-well plates. After 24 hours, cells were transfected at a final concentration of $50 \mathrm{nM}$ siRNA using Lipofectamine RNAiMAX (Life Technologies) according to the manufacturer's instructions. MTS assay (CellTiter 96 Aqueous One Solution Cell Proliferation Assay, Promega) was performed to assess the cell viability after 72 hours and 96 hours, according to the manufacturer's protocol.

\section{ACKNOWLEDGMENTS}

We have benefited from useful discussions with Mary C. Farach-Carson, J. N. Onuchic, and Donald S. Coffey.

\section{CONFLICTS OF INTEREST}

The authors declare no conflict of interest.

\section{GRANT SUPPORT}

This work was supported by the National Science Foundation (NSF) Center for Theoretical Biological Physics (NSF PHY-1427654) and NSF DMS-1361411. HL was also supported as a CPRIT (Cancer Prevention and Research Institute of Texas) Scholar in Cancer Research of the State of Texas at Rice University. EB-J was also supported by CPRIT and a grant from the Tauber Family Funds and the Maguy-Glass Chair in Physics of Complex Systems. SAM was supported by a grant from the National Institutes of Health (5RO1CA155243). SH was supported by the Rubenstein Family Foundation and the Canary Foundation. KJP was supported by National Cancer Institute (NCI) (Grant no. U54CA143803, CA163124, CA093900, and CA143055).

\section{REFERENCES}

1. Nieto MA. Epithelial plasticity: a common theme in embryonic and cancer cells. Science. 2013;342:1234850.

2. Kalluri R, Weinberg RA. The basics of epithelialmesenchymal transition. J Clin Invest. 2009;119:1420-8.

3. Revenu C, Gilmour D. EMT 2.0: shaping epithelia through collective migration. Curr Opin Genet Dev. 2009;19:33842.

4. Micalizzi DS, Farabaugh SM, Ford HL. Epithelialmesenchymal transition in cancer: parallels between normal development and tumor progression. J Mammary Gland Biol Neoplasia. 2010;15:117-34.

5. Jolly MK, Boareto M, Huang B, Jia D, Lu M, Ben-Jacob E, Onuchic JN, Levine H. Implications of the hybrid epithelial/ mesenchymal phenotype in metastasis. Front Oncol. 
2015;5:155.

6. Aceto N, Toner M, Maheswaran S, Haber DA. En Route to Metastasis: Circulating Tumor Cell Clusters and Epithelialto-Mesenchymal Transition. Trends in Cancer. 2015;1:4452.

7. Joosse SA, Gorges TM, Pantel K. Biology, detection, and clinical implications of circulating tumor cells. EMBO Mol Med. 2015;7:1-11.

8. Zheng X, Carstens JL, Kim J, Scheible M, Kaye J, Sugimoto H, Wu C-C, LeBleu VS, Kalluri R. Epithelialto-mesenchymal transition is dispensable for metastasis but induces chemoresistance in pancreatic cancer. Nature. 2015;527:525-30.

9. Fischer KR, Durrans A, Lee S, Sheng J, Li F, Wong STC, Choi H, El Rayes T, Ryu S, Troeger J, Schwabe RF, Vahdat LT, Altorki NK, et al. Epithelial-to-mesenchymal transition is not required for lung metastasis but contributes to chemoresistance. Nature. 2015;527:472-6.

10. Li W, Kang Y. Probing the Fifty Shades of EMT in Metastasis. Trends in Cancer. 2016;2:65-6.

11. Grosse-Wilde A, Fouquier d' Herouei A, McIntosh E, Ertaylan G, Skupin A, Kuestner RE, del Sol A, Walters K-A, Huang S. Stemness of the hybrid epithelial/ mesenchymal state in breast cancer and its association with poor survival. PLoS One. 2015;10:e126522.

12. Arnoux V, Côme C, Kusewitt DF, Hudson LG, Savagner P. Cutaneous Wound Reepithelialization. Rise and Fall of Epithelial Phenotype. Springer US; 2005. p. 111-34.

13. Savagner P. Epithelial-mesenchymal transitions: from cell plasticity to concept elasticity. Curr Top Dev Biol. 2015;112:273-300.

14. Watanabe K, Villarreal-Ponce A, Sun P, Salmans ML, Fallahi M, Andersen B, Dai X. Mammary morphogenesis and regeneration require the inhibition of EMT at terminal end buds by Ovol2 transcriptional repressor. Dev Cell. 2014;29:59-74.

15. Yaswen P. Reinforcing targeted therapeutics with phenotypic stability factors. Cell Cycle. 2015;13:3818-22.

16. Jia D, Jolly MK, Boareto M, Parsana P, Mooney SM, Pienta KJ, Levine H, Ben-Jacob E. OVOL guides the epithelialhybrid-mesenchymal transition. Oncotarget. 2015;6:1543648. doi: 10.18632/oncotarget.3623.

17. Hong $\mathrm{T}$, Watanabe $\mathrm{K}$, Ta $\mathrm{CH}$, Villarreal-Ponce A, Nie Q, Dai X. An Ovol2-Zeb1 Mutual Inhibitory Circuit Governs Bidirectional and Multi-step Transition between Epithelial and Mesenchymal States. PLOS Comput Biol. 2015;11:e1004569.

18. Jolly MK, Jia D, Boareto M, Mani SA, Pienta KJ, BenJacob E, Levine H. Coupling the modules of EMT and stemness: A tunable "stemness window" model. Oncotarget. 2015;6:25161-74. doi: 10.18632/oncotarget.4629.

19. Schliekelman MJ, Taguchi A, Zhu J, Dai X, Rodriguez J, Celiktas M, Zhang Q, Chin A, Wong C, Wang H, McFerrin L, Selemat S, Yang C, et al. Molecular portraits of epithelial, mesenchymal and hybrid states in lung adenocarcinoma and their relevance to survival. Cancer Res. 2015;75:1789-800.

20. Andriani F, Bertolini G, Facchinetti F, Baldoli E, Moro M, Casalini P, Caserini R, Milione M, Leone G, Pelosi G, Pastorino U, Sozzi G, Roz L. Conversion to stem-cell state in response to microenvironmental cues is regulated by balance between epithelial and mesenchymal features in lung cancer cells. Mol Oncol. 2016;10:253-71.

21. Jeevan DS, Cooper JB, Braun A, Murali RAJ, Jhanwaruniyal M. Molecular Pathways Mediating Metastases to the Brain via Epithelial-to-Mesenchymal Transition: Genes, Proteins, and Functional Analysis. Anticancer Res. 2016;36:523-32.

22. Yu M, Bardia A, Wittner BS, Stott SL, Smas ME, Ting DT, Isakoff SJ, Ciciliano JC, Wells MN, Shah AM, Concannon $\mathrm{KF}$, Donaldson MC, Sequist L V, et al. Circulating breast tumor cells exhibit dynamic changes in epithelial and mesenchymal composition. Science. 2013;339:580-4.

23. Bronsert P, Enderle-Ammour K, Bader M, Timme S, Kuehs M, Csanadi A, Kayser G, Kohler I, Bausch D, Hoeppner J, Hopt UT, Keck T, Stickeler E, et al. Cancer cell invasion and EMT marker expression: a three-dimensional study of the human cancer-host interface. J Pathol. 2014 Nov;234:410-22.

24. Aceto N, Bardia A, Miyamoto DT, Donaldson MC, Wittner BS, Spencer JA, Yu M, Pely A, Engstrom A, Zhu H, Brannigan BW, Kapur R, Stott SL, et al. Circulating tumor cell clusters are oligoclonal precursors of breast cancer metastasis. Cell. 2014;158:1110-22.

25. Brabletz S, Brabletz T. The ZEB/miR-200 feedback loop - a motor of cellular plasticity in development and cancer? EMBO Rep. 2010;11:670-7.

26. Werner S, Frey S, Riethdorf S, Schulze C, Alawi M, Kling L, Vafaizadeh V, Sauter G, Terracciano L, Schumacher U, Pantel K, Assmann V. Dual roles of the transcription factor grainyhead-like 2 (GRHL2) in breast cancer. J Biol Chem. 2013;288:22993-3008.

27. Varma S, Cao Y, Tagne JB, Lakshminarayanan M, Li J, Friedman TB, Morell RJ, Warburton D, Kotton DN, Ramirez MI. The transcription factors grainyhead-like 2 and NK2-homeobox 1 form a regulatory loop that coordinates lung epithelial cell morphogenesis and differentiation. J Biol Chem. 2012;287:37282-95.

28. Ren D, Wang M, Guo W, Huang S, Wang Z, Zhao X, Du H, Song L, Peng X. Double-negative feedback loop between ZEB2 and miR-145 regulates epithelial-mesenchymal transition and stem cell properties in prostate cancer cells. Cell Tissue Res. 2014;358:763-78.

29. Lu M, Jolly MK, Levine H, Onuchic JN, Ben-Jacob E. MicroRNA-based regulation of epithelial-hybridmesenchymal fate determination. Proc Natl Acad Sci U S A. $2013 ; 110: 18174-9$.

30. Roca H, Hernandez J, Weidner S, McEachin RC, Fuller 
D, Sud S, Schumann T, Wilkinson JE, Zaslavsky A, Li H, Maher CA, Daignault-Newton S, Healy PN, et al. Transcription Factors OVOL1 and OVOL2 Induce the Mesenchymal to Epithelial Transition in Human Cancer. PLoS One. 2013;8:e76773.

31. Peng X, Guo W, Liu T, Wang X, Tu X, Xiong D, Chen S, Lai Y, Du H, Chen G, Liu G, Tang Y, Huang S, et al. Identification of miRs-143 and -145 that is associated with bone metastasis of prostate cancer and involved in the regulation of EMT. PLoS One. 2011;6:e20341.

32. Hernandez JR, Kim JJ, Verdone JE, Liu X, Torga G, Pienta KJ, Mooney SM. Alternative CD44 splicing identifies epithelial prostate cancer cells from the mesenchymal counterparts. Med Oncol. 2015;32:159.

33. Aue A, Hinze C, Walentin K, Ruffert J, Yurtdas Y, Werth M, Chen W, Rabien A, Kilic E, Schulzke J-D, Schumann M, Schmidt-Ott KM. A Grainyhead-Like 2/Ovo-Like 2 Pathway Regulates Renal Epithelial Barrier Function and Lumen Expansion. J Am Soc Nephrol. 2015;26:2704-15.

34. Walentin K, Hinze C, Werth M, Haase N, Varma S, Morell R, Aue A, Pötschke E, Warburton D, Qiu A, Barasch J, Purfürst B, Dieterich C, et al. A Grhl2-dependent gene network controls trophoblast branching morphogenesis. Development. 2015;142:1125-36.

35. Kohn KW, Zeeberg BM, Reinhold WC, Pommier Y. Gene expression correlations in human cancer cell lines define molecular interaction networks for epithelial phenotype. PLoS One. 2014;9:e99269.

36. Park S-MM, Gaur AB, Lengyel E, Peter ME. The miR-200 family determines the epithelial phenotype of cancer cells by targeting the E-cadherin repressors ZEB1 and ZEB2. Genes Dev. 2008;22:894-907.

37. Ribeiro AS, Paredes J. P-Cadherin Linking Breast Cancer Stem Cells and Invasion: A Promising Marker to Identify an "Intermediate/Metastable" EMT State. Front Oncol. 2015;4:371.

38. Barretina J, Caponigro G, Stransky N, Venkatesan K, Margolin A a., Kim S, Wilson CJ, Lehár J, Kryukov G V., Sonkin D, Reddy A, Liu M, Murray L, et al. The Cancer Cell Line Encyclopedia enables predictive modelling of anticancer drug sensitivity. Nature. 2012;483:603-7.

39. Yang X, Vasudevan P, Parekh V, Penev A, Cunningham JM. Bridging cancer biology with the clinic: relative expression of a GRHL2-mediated gene-set pair predicts breast cancer metastasis. PLoS One. 2013;8:e56195.

40. Xiang X, Deng Z, Zhuang X, Ju S, Mu J, Jiang H, Zhang L, Yan J, Miller D, Zhang HG. Grhl2 Determines the Epithelial Phenotype of Breast Cancers and Promotes Tumor Progression. PLoS One. 2012;7:e50781.

41. Quan Y, Xu M, Cui P, Ye M, Zhuang B, Min Z. Grainyheadlike 2 promotes tumor growth and is associated with poor prognosis in colorectal cancer. J Cancer. 2015;6:342-50.

42. Ombrato L, Malanchi I. The EMT Universe: Space between Cancer Cell Dissemination and Metastasis Initiation. Crit
Rev Oncog. 2014;19:349-61.

43. Tam WL, Weinberg RA. The epigenetics of epithelialmesenchymal plasticity in cancer. Nat Med. 2013;19:143849.

44. Yang X, Lin X, Zhong X, Kaur S, Li N, Liang S, Lassus H, Wang L, Katsaros D, Montone K, Zhao X, Zhang Y, Bützow R, et al. Double-negative feedback loop between reprogramming factor LIN28 and microRNA let-7 regulates aldehyde dehydrogenase 1-positive cancer stem cells. Cancer Res. 2010;70:9463-72.

45. Jolly MK, Huang B, Lu M, Mani SA, Levine H, Ben-Jacob E. Towards elucidating the connection between epithelial mesenchymal transitions and stemness. J R Soc Interface. 2014;11:20140962.

46. Wilbert ML, Huelga SC, Kapeli K, Stark TJ, Liang TY, Chen SX, Yan BY, Nathanson JL, Hutt KR, Lovci MT, Kazan H, Vu AQ, Massirer KB, et al. LIN28 Binds Messenger RNAs at GGAGA Motifs and Regulates Splicing Factor Abundance. Mol Cell. 2012;48:195-206.

47. Zisoulis DG, Kai ZS, Chang RK, Pasquinelli AE. Autoregulation of microRNA biogenesis by let-7 and Argonaute. Nature. 2012;486:541-4.

48. Kong D, Banerjee S, Ahmad A, Li Y, Wang Z, Sethi $\mathrm{S}$, Sarkar FH. Epithelial to mesenchymal transition is mechanistically linked with stem cell signatures in prostate cancer cells. PLoS One. 2010;5:e12445.

49. Yong SL, Dutta A. The tumor suppressor microRNA let-7 represses the HMGA2 oncogene. Genes Dev. 2007;21:1025-30.

50. Tan EJ, Thuault S, Caja L, Carletti T, Heldin CH, Moustakas A. Regulation of transcription factor twist expression by the DNA architectural protein high mobility group A2 during epithelial-to-mesenchymal transition. J Biol Chem. 2012;287:7134-45.

51. Karwacki-Neisius V, Göke J, Osorno R, Halbritter F, Ng JH, Weiße AY, Wong FCK, Gagliardi A, Mullin NP, Festuccia N, Colby D, Tomlinson SR, Ng HH, et al. Reduced Oct4 expression directs a robust pluripotent state with distinct signaling activity and increased enhancer occupancy by Oct4 and Nanog. Cell Stem Cell. 2013;12:531-45.

52. Niwa H, Miyazaki J, Smith AG. Quantitative expression of Oct-3/4 defines differentiation, dedifferentiation or selfrenewal of ES cells. Nat Genet. 2000;24:372-6.

53. Theunissen TW, Van Oosten AL, Castelo-Branco G, Hall J, Smith A, Silva JCR. Nanog overcomes reprogramming barriers and induces pluripotency in minimal conditions. Curr Biol. 2011;21:65-71.

54. Shu J, Wu C, Wu Y, Li Z, Shao S, Zhao W, Tang X, Yang H, Shen L, Zuo X, Yang W, Shi Y, Chi X, et al. Induction of pluripotency in mouse somatic cells with lineage specifiers. Cell. 2013;153:963-75.

55. Chickarmane V, Olariu V, Peterson C. Probing the role of stochasticity in a model of the embryonic stem cell - heterogeneous gene expression and reprogramming 
efficiency. BMC Syst Biol. 2012;6:98.

56. Liu S, Cong Y, Wang D, Sun Y, Deng L, Liu Y, MartinTrevino R, Shang L, McDermott SP, Landis MD, Hong S, Adams A, D'Angelo R, et al. Breast cancer stem cells transition between epithelial and mesenchymal states reflective of their normal counterparts. Stem Cell Reports. 2014;2:78-91.

57. Quan Y, Jin R, Huang A, Zhao H, Feng B, Zang L, Zheng M. Downregulation of GRHL2 inhibits the proliferation of colorectal cancer cells by targeting ZEB1. Cancer Biol Ther. 2014; 15:878-87.

58. Polytarchou C, Iliopoulos D, Struhl K. An integrated transcriptional regulatory circuit that reinforces the breast cancer stem cell state. Proc Natl Acad Sci U S A. 2012;109:14470-5.

59. Cieply B, Farris J, Denvir J, Ford HL, Frisch SM. Epithelial-Mesenchymal Transition and Tumor Suppression Are Controlled by a Reciprocal Feedback Loop between ZEB1 and Grainyhead-like-2. Cancer Res. 2013;73:6299309.

60. Cieply B, Riley IV P, Pifer PM, Widmeyer J, Addison JB, Ivanov A V., Denvir J, Frisch SM. Suppression of the epithelial-mesenchymal transition by grainyhead-like-2. Cancer Res. 2012;72:2440-53.

61. Mehrazarin S, Chen W, Oh J-E, Liu ZX, Kang KL, Yi JK, Kim RH, Shin K-H, Park N-H, Kang MK. p63 Gene is Regulated by Grainyhead-Like 2 (GRHL2) Through Reciprocal Feedback and Determines Epithelial Phenotype in Human Keratinocytes. J Biol Chem. 2015;290:1999920008.

62. Lee B, Villarreal-Ponce A, Fallahi M, Ovadia J, Sun P, Yu Q-C, Ito S, Sinha S, Nie Q, Dai X. Transcriptional mechanisms link epithelial plasticity to adhesion and differentiation of epidermal progenitor cells. Dev Cell. 2014;29:47-58.

63. Steinway SN, Gomez Tejeda Zañudo J, Ding W, Rountree CB, Feith DJ, Loughran TP, Albert R. Network modeling of TGF signaling in hepatocellular carcinoma epithelial-tomesenchymal transition reveals joint Sonic hedgehog and Wnt pathway activation. Cancer Res. 2014;74:5963-77.

64. Steinway SN, Zañudo JGT, Michel PJ, Feith DJ, Loughran TP, Albert R. Combinatorial interventions inhibit TGF-driven epithelial-to-mesenchymal transition and support hybrid cellular phenotypes. npj Syst Biol Appl. 2015;1:15014.

65. Sampson VB, David JM, Puig I, Patil PU, de Herreros AG, Thomas G V., Rajasekaran AK. Wilms' tumor protein induces an epithelial-mesenchymal hybrid differentiation state in clear cell renal cell carcinoma. PLoS One. 2014;9:e102041.

66. Huang RY-J, Wong MK, Tan TZ, Kuay KT, Ng a HC, Chung VY, Chu Y-S, Matsumura N, Lai H-C, Lee YF, Sim W-J, Chai C, Pietschmann E, et al. An EMT spectrum defines an anoikis-resistant and spheroidogenic intermediate mesenchymal state that is sensitive to e-cadherin restoration by a src-kinase inhibitor, saracatinib (AZD0530). Cell Death Dis. 2013;4:e915.

67. Zuo J, Ishikawa T, Boutros S, Xiao Z, Humtsoe JO, Kramer RH. Bcl-2 overexpression induces a partial epithelial to mesenchymal transition and promotes squamous carcinoma cell invasion and metastasis. Mol Cancer Res. 2010;8:17082.

68. Umbreit C, Flanjak J, Weiss C, Erben P, Aderhold C, Faber A, Stern-straeter J, Hoermann K, Schultz JD. Incomplete Epithelial - Mesenchymal Transition in p16-positive Squamous Cell Carcinoma Cells Correlates with -Catenin Expression. Anticancer Res. 2014;34:7061-70.

69. Klymkowsky MW, Savagner P. Epithelial-mesenchymal transition: a cancer researcher's conceptual friend and foe. Am J Pathol. 2009;174:1588-93.

70. Leroy P, Mostov KE. Slug Is Required for Cell Survival during Partial Epithelial-Mesenchymal Transition of HGFinduced tubulogenesis. J Cell Sci. 2007;18:1943-52.

71. Leopold PL, Vincent J, Wang H. A comparison of epithelialto-mesenchymal transition and re-epithelialization. Semin Cancer Biol. 2012;22(5-6):471-83.

72. Abell AN, Jordan NV, Huang W, Prat A, Midland AA, Johnson NL, Granger D a, Mieczkowski PA, Perou CM, Gomez SM, Li L, Johnson GL. MAP3K4/CBP-regulated $\mathrm{H} 2 \mathrm{~B}$ acetylation controls epithelial-mesenchymal transition in trophoblast stem cells. Cell Stem Cell. 2011;8:525-37.

73. Lecharpentier A, Vielh P, Perez-Moreno P, Planchard D, Soria JC, Farace F. Detection of circulating tumour cells with a hybrid (epithelial/mesenchymal) phenotype in patients with metastatic non-small cell lung cancer. Br J Cancer. 2011;105:1338-41.

74. Bovier A. Metastability: a potential theoretic approach. Proceedings of the Inernational Congress of Mathematicians. 2006. p. 499-518.

75. Alon U. Network motifs: theory and experimental approaches. Nat Rev Genet. 2007;8:450-61.

76. Dang TT, Esparza MA, Maine EA, Westcott JM, Pearson GW. Np63 promotes breast cancer cell motility through the selective activation of components of the Epithelialto-Mesenchymal Transition program. Cancer Res. 2015;75:3925-35.

77. Swetha G, Chandra V, Phadnis S, Bhonde R. Glomerular parietal epithelial cells of adult murine kidney undergo EMT to generate cells with traits of renal progenitors. J Cell Mol Med. 2011;15:396-413.

78. Yovchev MI, Grozdanov PN, Zhou H, Racherla H, Guha C, Dabeva MD. Identification of adult hepatic progenitor cells capable of repopulating injured rat liver. Hepatology. 2008;47:636-47.

79. Biddle A, Liang X, Gammon L, Fazil B, Harper LJ, Emich H, Costea DE, Mackenzie IC. Cancer stem cells in squamous cell carcinoma switch between two distinct phenotypes that are preferentially migratory or proliferative. 
Cancer Res. 2011;71:5317-26.

80. Strauss R, Li Z-Y, Liu Y, Beyer I, Persson J, Sova P, Möller T, Pesonen S, Hemminki A, Hamerlik P, Drescher C, Urban $\mathrm{N}$, Bartek J, et al. Analysis of epithelial and mesenchymal markers in ovarian cancer reveals phenotypic heterogeneity and plasticity. PLoS One. 2011;6:e16186.

81. Sarrio D, Rodriguez-Pinilla SM, Hardisson D, Cano A, Moreno-Bueno G, Palacios J. Epithelial-mesenchymal transition in breast cancer relates to the basal-like phenotype. Cancer Res. 2008;68:989-97.

82. Strauss R, Sova P, Liu Y, Zong YL, Tuve S, Pritchard D, Brinkkoetter P, Möller T, Wildner O, Pesonen S, Hemminki A, Urban N, Drescher C, et al. Epithelial phenotype confers resistance of ovarian cancer cells to oncolytic adenoviruses. Cancer Res. 2009;69:5115-25.

83. Schmidt JM, Panzilius E, Bartsch HS, Irmler M, Beckers J, Kari V, Linnemann JR, Dragoi D, Hirschi B, Kloos UJ, Sass S, Theis F, Kahlert S, et al. Stem-Cell-like Properties and Epithelial Plasticity Arise as Stable Traits after Transient Twist1 Activation. Cell Rep. 2015;10:131-9.

84. Tran HD, Luitel K, Kim M, Zhang K, Longmore GD, Tran DD. Transient SNAIL1 expression is necessary for metastatic competence in breast cancer. Cancer Res. 2014;74:6330-40.

85. Goldman A, Majumder B, Dhawan A, Ravi S, Goldman D, Kohandel M, Majumder PK, Sengupta S. Temporally sequenced anticancer drugs overcome adaptive resistance by targeting a vulnerable chemotherapy-induced phenotypic transition. Nat Commun. 2015;6:6139.

86. Guan J, Zhang H, Wen Z, Gu Y, Cheng Y, Sun Y, Zhang T, Jia C, Lu Z, Chen J. Retinoic acid inhibits pancreatic cancer cell migration and EMT through the downregulation of IL-6 in cancer associated fibroblast cells. Cancer Lett. 2014;345:132-9.

87. Biddle A, Gammon L, Liang X, Costea DE, Mackenzie IC. Phenotypic Plasticity Determines Cancer Stem Cell Therapeutic Resistance in Oral Squamous Cell Carcinoma. EBioMedicine. 2016;4:138-45.

88. Ye X, Leong Tam W, Shibue T, Kaygusuz Y, Reinhardt F, $\mathrm{Ng}$ Eaton E, Weinberg RA. Distinct EMT programs control normal mammary stem cells and tumour-initiating cells. Nature. 2015;525:256-60.

89. Ganesan R, Mallets E, Gomez-Cambronero J. The transcription factors Slug (SNAI2) and Snail (SNAI1) regulate phospholipase $\mathrm{D}$ (PLD) promoter in opposite ways towards cancer cell invasion. Mol Oncol. 2015; in press.

90. Chung VY, Tan TZ, Tan M, Wong MK, Kuay KT, Yang Z, Ye J, Muller J, Koh CM, Guccione E, Thiery JP, Huang RY-J. GRHL2-miR-200-ZEB1 maintains the epithelial status of ovarian cancer through transcriptional regulation and histone modification. Sci Rep. 2016;6:19943.

91. Grande MT, Sánchez-Laorden B, López-Blau C, De Frutos CA, Boutet A, Arévalo M, Rowe RG, Weiss SJ, López-
Novoa JM, Nieto MA. Snaill-induced partial epithelial-tomesenchymal transition drives renal fibrosis in mice and can be targeted to reverse established disease. Nat Med. 2015;21:989-97.

92. Morbini $\mathrm{P}$, Inghilleri S, Campo I, Oggionni T, Zorzetto M, Luisetti M. Incomplete expression of epithelialmesenchymal transition markers in idiopathic pulmonary fibrosis. Pathol Res Pract. 2011;207:559-67.

93. Tan TZ, Miow QH, Miki Y, Noda T, Mori S, Huang RY, Thiery JP. Epithelial-mesenchymal transition spectrum quantification and its efficacy in deciphering survival and drug responses of cancer patients. EMBO Mol Med. 2014;6:1279-93.

94. Lundgren K, Nordenskjöld B, Landberg G. Hypoxia, Snail and incomplete epithelial-mesenchymal transition in breast cancer. Br J Cancer. 2009;101:1769-81.

95. Tian X-J, Zhang H, Xing J. Coupled Reversible and Irreversible Bistable Switches Underlying TGF-induced Epithelial to Mesenchymal Transition. Biophys J. 2013;105:1079-89.

96. Zhang J, Tian X-J, Zhang H, Teng Y, Li R, Bai F, Elankumaran S, Xing J. TGF--induced epithelial-tomesenchymal transition proceeds through stepwise activation of multiple feedback loops. Sci Signal. 2014;7:ra91.

97. Taube JH, Herschkowitz JI, Komurov K, Zhou AY, Gupta S, Yang J, Hartwell K, Onder TT, Gupta PB, Evans KW, Hollier BG, Ram PT, Lander ES, et al. Core epithelialto-mesenchymal transition interactome gene-expression signature is associated with claudin-low and metaplastic breast cancer subtypes. Proc Natl Acad Sci U S A. 2010;107:15449-54.

98. Hurteau GJ, Carlson JA, Roos E, Brock GJ. Stable expression of miR-200c alone is sufficient to regulate TCF8 (ZEB1) and restore E-cadherin expression. Cell Cycle. 2009;8:2064-9.

99. Cohen DPA, Martignetti L, Robine S, Barillot E, Zinovyev A, Calzone L. Mathematical Modelling of Molecular Pathways Enabling Tumour Cell Invasion and Migration. PLOS Comput Biol. 2015;11:e1004571.

100. Sarioglu AF, Aceto N, Kojic N, Donaldson MC, Zeinali M, Hamza B, Engstrom A, Zhu H, Sundaresan TK, Miyamoto DT, Luo X, Bardia A, Wittner BS, et al. A microfluidic device for label-free, physical capture of circulating tumor cell clusters. Nat Methods. 2015;12:685-91.

101. De Craene B, Berx G. Regulatory networks defining EMT during cancer initiation and progression. Nat Rev Cancer. 2013;13:97-110.

102. Lee J, Lee J, Farquhar KS, Yun J, Frankenberger CA, Bevilacqua E, Yeung K, Kim E-J, Balázsi G, Rosner MR. Network of mutually repressive metastasis regulators can promote cell heterogeneity and metastatic transitions. Proc Natl Acad Sci U S A. 2014;111:E364-73. 
103. Huang B, Lu M, Jolly MK, Tsarfaty I, Onuchic J, Ben-Jacob E. The three-way switch operation of Rac1/RhoA GTPasebased circuit controlling amoeboid-hybrid-mesenchymal transition. Sci Rep. 2014;4:6449.

104. Huang B, Jolly MK, Lu M, Tsarfaty I, Onuchic JN, BenJacob E. Modeling the transitions between collective and solitary migration phenotypes in cancer metastasis. Sci Rep. 2015;5:17379.

105. Milo R, Jorgensen P, Moran U, Weber G, Springer M. BioNumbers - the database of key numbers in molecular and cell biology. Nucleic Acids Res. 2010 Jan;38(Database issue):D750-3.
106. Schwanhäusser B, Busse D, Li N, Dittmar G, Schuchhardt J, Wolf J, Chen W, Selbach M. Global quantification of mammalian gene expression control. Nature. 2011;473:33742.

107. Lim LP, Lau NC, Weinstein EG, Abdelhakim A, Yekta S, Rhoades MW, Burge CB, Bartel DP. The microRNAs of Caenorhabditis elegans. Genes Dev. 2003;17:991-1008.

108. Goswami CP, Nakshatri H. PROGgene: gene expression based survival analysis web application for multiple cancers. J Clin Bioinforma. 2013;3:22. 